\title{
Suppression of the SLC7A11/glutathione axis causes synthetic lethality in KRAS-mutant lung adenocarcinoma
}

\author{
Kewen Hu, ${ }^{1,2}$ Kun Li, ${ }^{1}$ Jing Lv, ${ }^{1}$ Jie Feng, ${ }^{3}$ Jing Chen, ${ }^{4}$ Haigang Wu, ${ }^{1}$ Feixiong Cheng, $, 5,6,7$ Wenhao Jiang, ${ }^{1}$ Jieqiong Wang, ${ }^{2}$ \\ Haixiang Pei,, ${ }^{1}$ Paul J. Chiao, ${ }^{8}$ Zhenyu Cai, ${ }^{9}$ Yihua Chen, ${ }^{1}$ Mingyao Liu, ${ }^{1}$ and Xiufeng Pang ${ }^{1}$ \\ 'Shanghai Key Laboratory of Regulatory Biology, Institute of Biomedical Sciences and School of Life Sciences, East China Normal University, Shanghai, China. ${ }^{2}$ Cancer Institute, Fudan University Shanghai \\ Cancer Center, Department of Oncology, Shanghai Medical College, Fudan University, Shanghai, China. 'Beijing Neurosurgical Institute, Beijing Tiantan Hospital, Capital Medical University, Beijing, China. \\ ${ }^{4}$ Key Laboratory of Reproduction and Genetics in Ningxia, Ningxia Medical University, Yinchuan, China. ${ }^{5}$ Cenomic Medicine Institute, Lerner Research Institute, Cleveland Clinic, Cleveland, Ohio, USA. \\ ${ }^{6}$ Department of Molecular Medicine, Cleveland Clinic Lerner College of Medicine, Case Western Reserve University, Cleveland, Ohio, USA. Case Comprehensive Cancer Center, Case Western Reserve University \\ School of Medicine, Cleveland, Ohio, USA. ${ }^{8}$ Department of Molecular and Cellular Oncology, The University of Texas MD Anderson Cancer Center, Houston, Texas, USA. ${ }^{9}$ National Center for Liver Cancer, \\ Eastern Hepatobiliary Surgery Hospital, Second Military Medical University, Shanghai, China.
}

\begin{abstract}
Oncogenic KRAS is a major driver in lung adenocarcinoma (LUAD) that has yet to be therapeutically conquered. Here we report that the SLC7A11/glutathione axis displays metabolic synthetic lethality with oncogenic KRAS. Through metabolomics approaches, we found that mutationally activated KRAS strikingly increased intracellular cystine levels and glutathione biosynthesis. SLC7A11, a cystine/glutamate antiporter conferring specificity for cystine uptake, was overexpressed in patients with KRAS-mutant LUAD and showed positive association with tumor progression. Furthermore, SLC7A11 inhibition by either genetic depletion or pharmacological inhibition with sulfasalazine resulted in selective killing across a panel of KRASmutant cancer cells in vitro and tumor growth inhibition in vivo, suggesting the functionality and specificity of SLC7A11 as a therapeutic target. Importantly, we further identified a potent SLC7A11 inhibitor, HC106, that markedly decreased cystine uptake and intracellular glutathione biosynthesis. Furthermore, HG106 exhibited selective cytotoxicity toward KRAS-mutant cells by increasing oxidative stress- and ER stress-mediated cell apoptosis. Of note, treatment of KRAS-mutant LUAD with HC106 in several preclinical lung cancer mouse models led to marked tumor suppression and prolonged survival. Overall, our findings reveal that KRAS-mutant LUAD cells are vulnerable to SLC7A11 inhibition, offering potential therapeutic approaches for this currently incurable disease.
\end{abstract}

\section{Introduction}

Lung cancer is the leading cause of global cancer-related mortality, and lung adenocarcinoma (LUAD) represents the most common histological subtype of this disease. Approximately two-thirds of LUADs have activated oncogenes, such as EGFR, KRAS, translocated $A L K, R E T$, or ROS1. Among them, mutations in KRAS occur in nearly $30 \%$ of all lung cancer cases. Oncogenic KRAS mutations frequently compromise its GTPase activity, locking the protein in a persistent GTP-bound state and activating downstream signaling pathways, ultimately initiating lethal malignancies, including LUAD (1). Molecular targeted therapies have significantly improved the survival of patients with a therapeutically targetable lesion compared with conventional chemotherapy $(2,3)$. However, no therapeutic agent directly targeting RAS has been clinically approved $(4,5)$. Strategies to inhibit KRAS have been hindered by

Authorship note: $\mathrm{KH}, \mathrm{KL}$, and JL are co-first authors.

Conflict of interest: The authors have declared that no conflict of interest exists. Copyright: @ 2020, American Society for Clinical Investigation.

Submitted: August 9, 2018; Accepted: December 19, 2019; Published: March 3, 2020.

Reference information: / Clin Invest. 2020;130(4):1752-1766.

https://doi.org/10.1172/JCl124049. the lack of a proper binding pocket for small molecules. Recently, an atropisomeric inhibitor directly targeting mutant KRAS(G12C), a specific mutation in $11 \%-16 \%$ of LUADs, has been reported to achieve benefits in mice and in clinical trials (6-9). However, its action in patients still needs to be determined. Strategies to target KRAS downstream effectors showed modest or no clinical responses in KRAS-mutant LUAD either through single agents or in combination with chemotherapy (10-12). This can be explained by the reactivation of upstream kinases, alternative pathway effectors, KRAS dimerization, or other intrinsic and adaptive resistance mechanisms $(10,13)$. Therefore, it is imperative that alternative options for KRAS-mutant LUAD be developed.

KRAS mutations are frequently associated with a metabolic reprogramming, such as highly active glucose metabolism, differential channeling of glucose intermediates, reprogrammed glutamine metabolism, and increased autophagy and macropinocytosis $(4,14)$. These changes are critical for utilization of nutrients to satisfy biosynthetic demands and maintain redox equilibrium for cell survival. In order to support their growth, cells harboring KRAS mutations may undergo oncogenic stress to which cancer cells have to adapt. As a result, KRAS-mutant cancers become highly dependent on their metabolic adaptations, thereby expos- 
ing their Achilles' heel $(15,16)$. In cancer cells overexpressing specific oncogenes that are difficult to target directly, such as mutant KRAS, silencing their synthetic lethal partners would lead to specific elimination of these cells without harming normal cells. Emerging evidence implies that KRAS-mutant cancers are vulnerable to inhibition of their aberrant metabolism, and metabolic synthetic lethality can be an ideal target $(17,18)$.

In this study, we investigated metabolic networks in paired WT and mutant KRAS isogenic cells through metabolomics analysis, aiming to unravel metabolic vulnerabilities for the treatment of KRAS-mutant LUAD. This work identified solute carrier family 7 member 11 (SLC7A11; also known as XCT) as a protein with altered expression upon KRAS mutation, resulting in rewiring of glutathione metabolism. SLC7A11 is the functional light chain subunit of the cystine/glutamate antiporter system $\mathrm{x}_{c}^{-}$and confers specificity for cystine uptake, a major rate-limiting factor in glutathione biosynthesis (19). SLC7A11 is overexpressed in several cancer types, including glioma, liver carcinoma, non-small cell lung cancer, and triple-negative breast cancer, and serves as an independent prognostic factor (20-24). SLC7A11 also contributes to glutathione-based drug resistance (25-27). However, the specific role and therapeutic implications of SLC7A11 induction in $K R A S$-mutant LUAD remain elusive. Here we systemically investigated the synthetic lethal role of SLC7A11 in KRAS-transformed cells in vitro and in vivo. By chemical screening, we further identified the molecule HG106 as a potent SLC7A11 inhibitor. Inhibition of SLC7A11 by HG106 led to enhanced ROS generation, ER stress, and ultimately growth arrest specifically in KRAS-mutant LUAD.

\section{Results}

Mutant KRAS drives reprogramming of glutathione metabolism. To explore metabolic vulnerability in cancer cells driven by KRAS mutations, we obtained isogenic human pancreatic epithelial nestin-expressing (HPNE) cells with or without expression of constitutively active mutant KRAS(G12V) (28). We performed gas chromatography with TOF-mass spectrometry-based (GC/ TOF-MS-based) metabolomics analysis. Hierarchical clustering and heatmap analysis revealed that HPNE and HPNE/KRAS were grouped into distinct metabolite clusters, implying a marked difference in their metabolic signatures (Figure 1A and Supplemental Table 1; supplemental material available online with this article; https://doi.org/10.1172/JCI124049DS1). The pathway enrichment of those significantly changed metabolites further demonstrated that many metabolic pathways were increased in $K R A S$-mutant cells, with the glutathione metabolism pathway most significantly affected (Figure 1B and Supplemental Table 2). Although the arginine and proline metabolism pathway was enriched comparably to the glutathione metabolism pathway in our experimental system, we could not deduce any mechanistic explanation for the role of arginine and proline metabolism in $K R A S$-mutant lung cancer from the existing literature. Since glutathione metabolism has shown an emerging role in the growth of KRAS-driven cancers (29-32), we concentrated on this metabolic pathway for the mechanism study and inhibitor identification. As indicated in Figure 1C, the critical intermediates of glutathione metabolism in HPNE/KRAS cells were significantly upregulated, including cystine, glutamate, and glutathione. Cystine, a major rate-limiting factor in the process of glutathione biosynthesis, is imported by the cystine/glutamate antiporter system $\mathrm{x}_{\mathrm{c}}{ }^{-}$from the extracellular environment. Hence, we quantified cystine levels in two paired isogenic cell lines that were selected based on the dependence of growth and viability on mutant KRAS status. As expected, both KRAS-mutant HPNE (HPNE/KRAS) and H522 (H522/KRAS) cells exhibited much higher levels of $\mathrm{Na}^{+}$-independent $\left[{ }^{14} \mathrm{C}\right]$-cystine uptake, accompanied by a remarkable increase in glutathione biosynthesis, compared with their WT counterparts (Figure 1D). As a consequence of glutathione upregulation, KRAS-mutant cells generated more ROS to maintain redox equilibrium (Figure 1D). This is in accordance with previous studies showing that abundant ROS production is one of the characteristics of RAS activation $(31,33)$. These data collectively suggest that $K R A S$-mutant cells sustain highly active glutathione biosynthesis by absorbing more cystine from the extracellular environment.

SLC7A11 is overexpressed in KRAS-mutant LUAD. Given that $K R A S$-mutant cells take up more cystine, we set out to determine whether SLC7A11, a specific and functional subunit of system $\mathrm{x}_{\mathrm{c}}^{-}$, is the dominant factor of this biological process. By using paired KRAS isogenic lung and pancreatic cell lines, we found that mutant KRAS upregulated SLC7A11 mRNA levels (Figure 2A). The transcription level of NF-E2-related factor 2 (Nrf2; encoded by NRF2, also known as NFE2L2), a specific transcription factor of SLC7A11 (34), was coincidently upregulated in HPNE/KRAS and H522/ KRAS cells (Figure 2A). Moreover, the protein level of SLC7A11 and Nrf2 was elevated by introduction of KRAS mutations (Figure 2B). To further support these findings, we examined SLC7A11 protein expression in mouse lung tumors. Our Western blot analysis (Figure 2C) showed that there was an increase in SLC7A11 expression in lung tumor tissue compared with adjacent normal tissue in LSL-Kras ${ }^{\mathrm{G} 12 \mathrm{D}}$ transgenic mice 4 months after induction by intratracheal delivery of adenovirus-Cre. Similar results were additionally observed in isolated mouse embryonic fibroblasts (Figure 2D). These data demonstrate a positive relationship between SLC7A11 expression and KRAS mutational status. To investigate the mechanism of Nrf2 regulation by oncogenic KRAS, we silenced KRAS in KRAS-mutant A549 and H441 cells, and found that Nrf2 and SLC7A11 protein levels were coincidently decreased (Figure 2E), implying that Nrf2 might be a target of KRAS. To understand the mechanisms of SCL7A11 upregulation by Nrf2, we generated Nrf2-KO A549 and H441 cell lines via CRISPR/Cas9. Our results showed that genetic disruption of Nrf2 led to SLC7A11 suppression (Figure 2F), implying that Nrf2 mediated SLC7A11 expression in the setting of KRAS mutations. To identify KRAS effectors responsible for Nrf2 and SLC7A11 expression, we further used several pharmacological inhibitors to specifically block the main downstream pathways of KRAS, including the MAPK pathway, the PI3K/AKT pathway, and the Ral pathway, in A549 (Figure 2, G and $\mathrm{H})$ and H441 cells (Supplemental Figure 1, A and B). Our results showed that blockade of the key components downstream of KRAS decreased SLC7A11 and Nrf2 expression at both the mRNA and protein levels. These results suggest that key pathways downstream of KRAS share the ability to modulate the Nrf2/SLC7A11 axis, implying a cooperative regulatory mechanism.

In parallel, we investigated the clinical implications of SLC7A11 in patients with LUAD harboring KRAS mutations. We collected 
A Class

HPNE

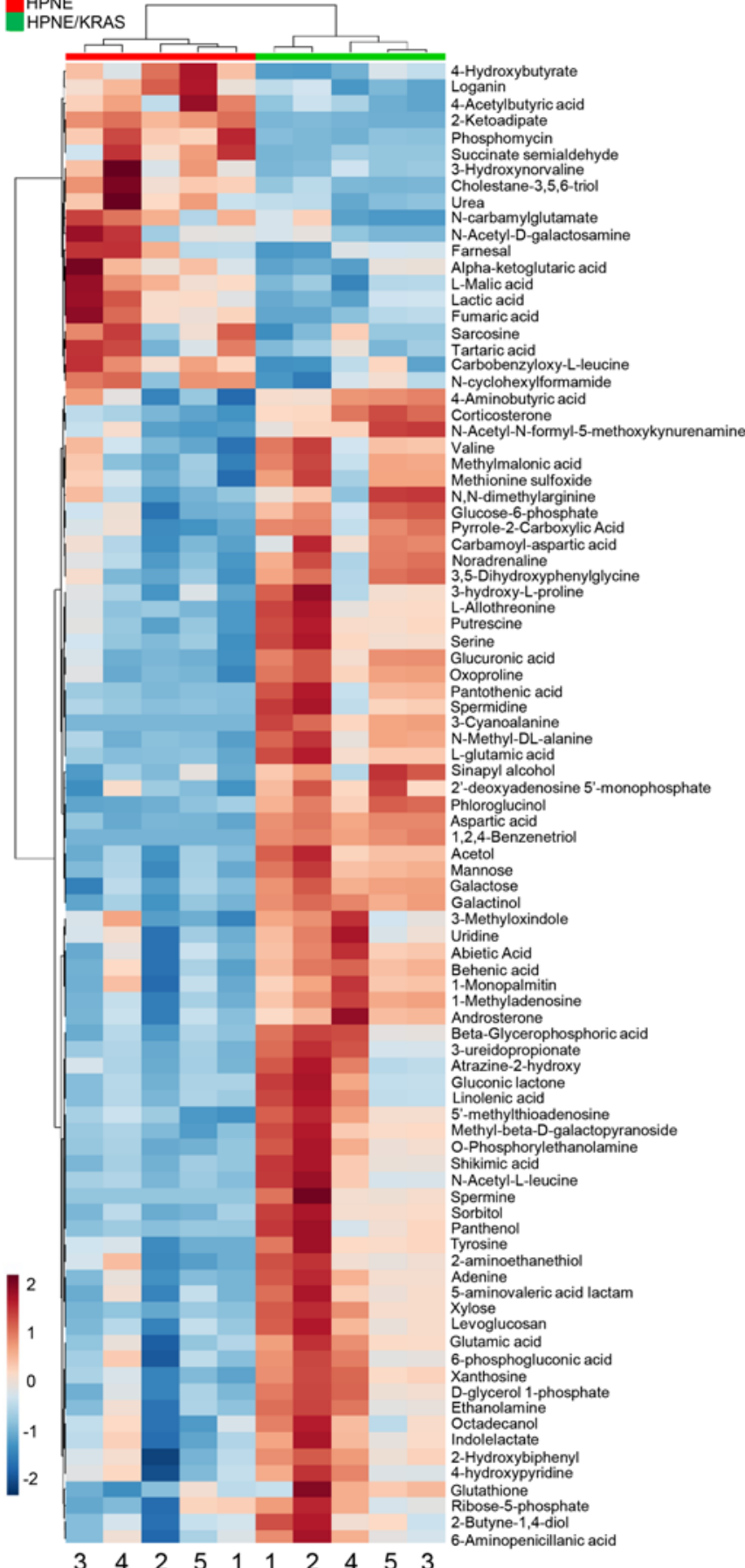

B
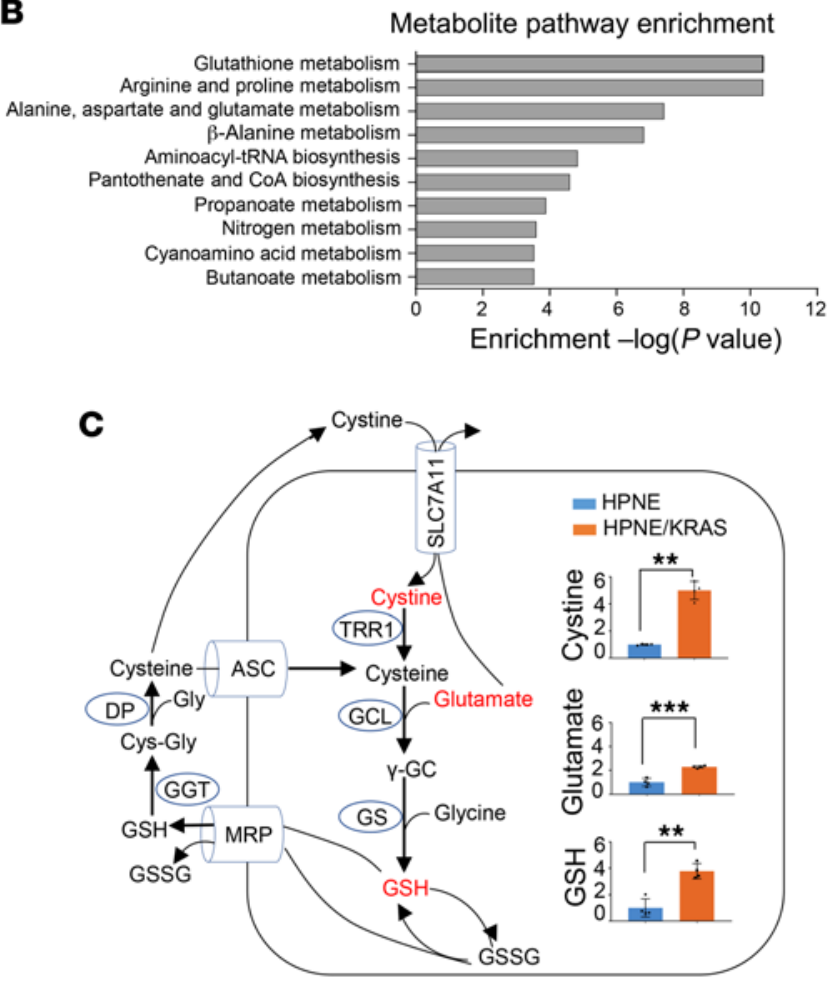

D
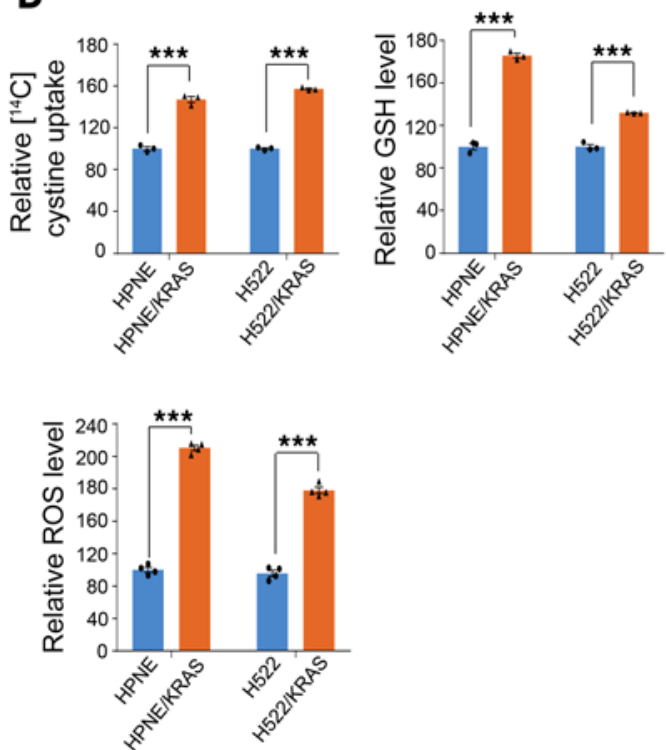

Figure 1. Mutant KRAS drives glutathione metabolism reprogramming. (A) Heatmap showing significantly differently expressed metabolites $(P<0.05)$ between HPNE and HPNE/KRAS groups. Values are scaled as indicated $(2$ to -2$)(n=5)$. (B) The top 10 enriched pathways from integrated pathway analysis of significantly changed metabolites. The $P$ value cutoff was 0.05 and represents the significance of enrichment of the pathway. (C) Illustration of the GSH metabolism pathway (left) and the relative levels of cystine, glutamate, and glutathione (GSH; right) $(n=5)$. ASC, alanine-serine-cysteine transporter; DP, dipeptidase; GGT, $\gamma$-glutamyl transpeptidase; GSSG, glutathione disulfide; MRP, multidrug resistance-associated protein; TRR1, thioredoxin reductase 1; GCL, glutamate-cysteine ligase; GS, glutamine synthetase; $\gamma$-GC, $\gamma$-glutamylcysteine. (D) Cystine, GSH, and ROS levels quantified in KRAS isogenic cell lines. $\mathrm{Na}^{+}$-independent $\left[{ }^{14} \mathrm{C}\right]$-cystine uptake was analyzed by a scintillation counter. The intracellular GSH content was measured using a GSH/CSSG-Glo assay kit. For the determination of ROS production, the cells were loaded with DCFH-DA, and fluorescence intensity was measured by flow cytometry. The levels in KRAS WT cells were defined as $100 \%$. Results shown are representative of 3 independent experiments. Data are represented as mean \pm SD of biological triplicates. ${ }^{* *} P<0.01,{ }^{* *} P<0.001$ by unpaired, 2 -tailed Student's $t$ tests. 


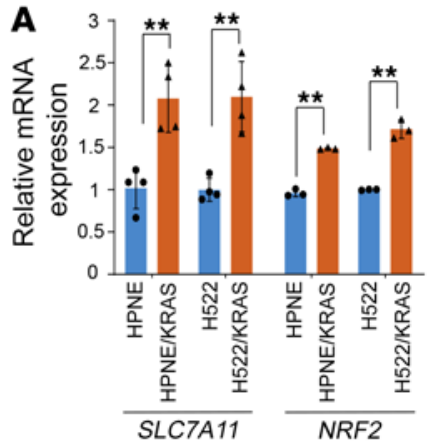

B

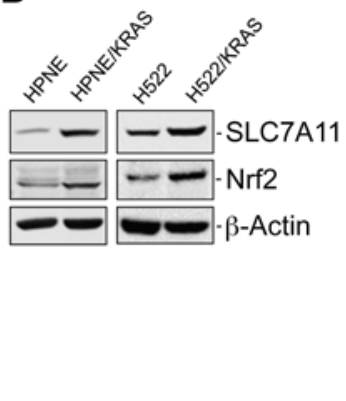

C

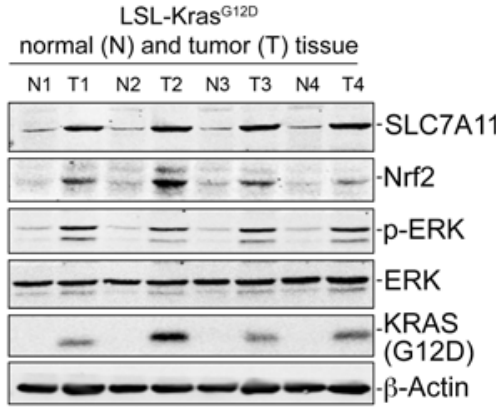

F $\quad$ A549 H441 KRAS(G12S) KRAS(G12V) KRAS(G12S) $\quad$ KRAS(G12V)
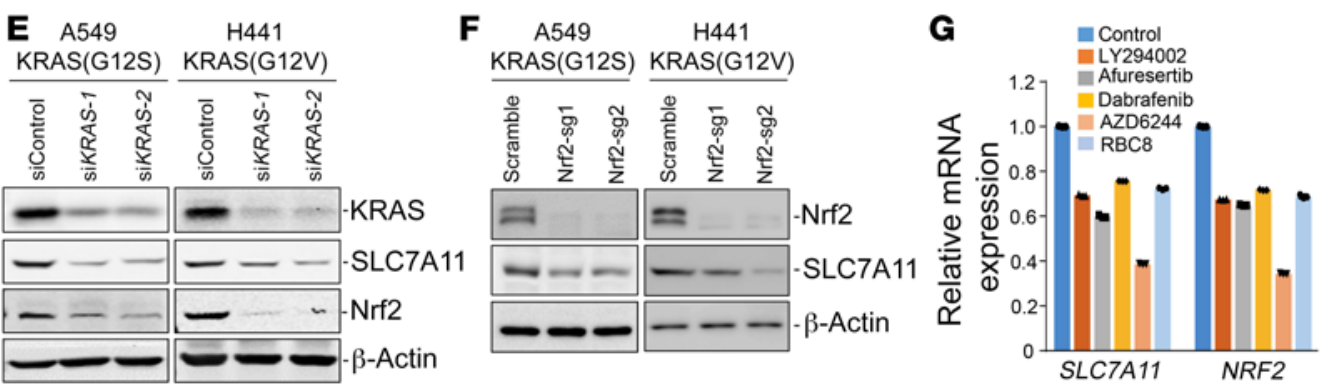

D

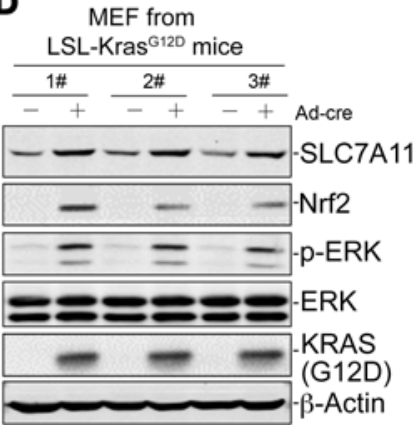

\section{H}

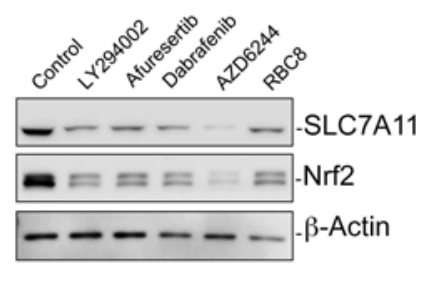

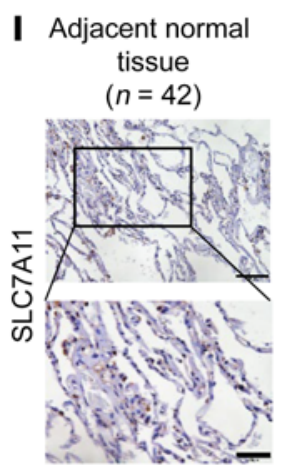
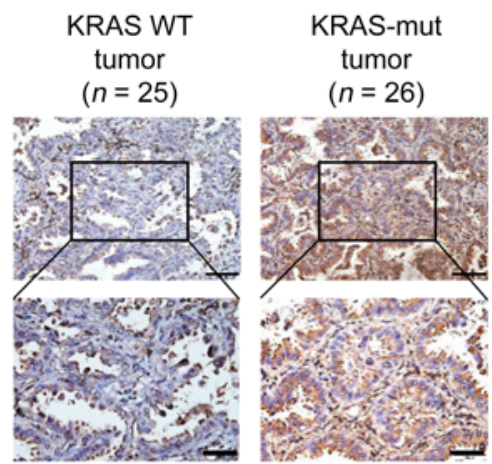
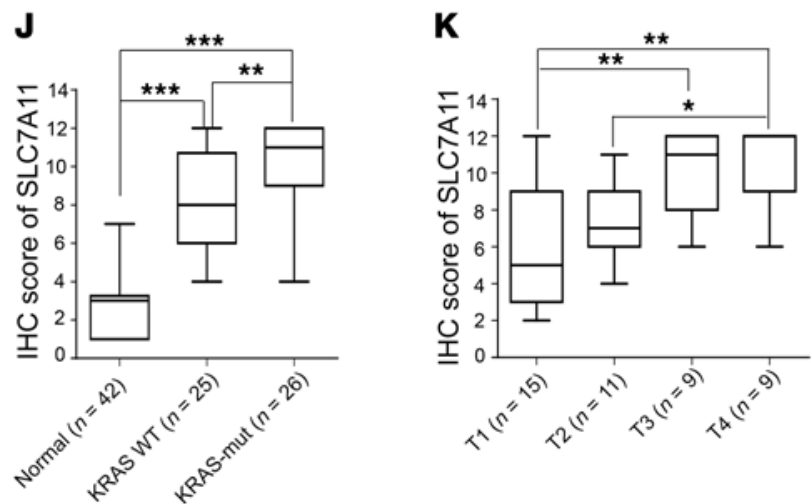

Figure 2. SLC7A11 is overexpressed in KRAS-mutant LUAD. (A) mRNA levels of SLC7A11 and NRF2. (B) Protein levels of SLC7A11 and Nrf2. For each cell pair, SLC7A11 and Nrf2 are contemporaneous immunoblots that run in parallel from the same biological replicate. (C) SLC7A11 and Nrf2 expression in normal lung tissues $(\mathrm{N})$ and tumors $(\mathrm{T})$ from LSL-Kras ${ }^{\mathrm{G120}}$ mice $(n=4)$. p-ERK was stripped, and the same membrane was then immunoblotted for total ERK. (D) Nrf2 and SLC7A11 expression in mouse embryonic fibroblasts (MEFs). MEFs were generated from LSL-Kras ${ }^{\mathrm{G} 120}$ mice and induced by adenovirus-Cre (Ad-cre) for 48 hours $(n=3)$. p-ERK and total ERK are contemporaneous immunoblots that run in parallel from the same biological replicate. (E) Nrf2 and SLC7A11 expression upon KRAS silencing. For individual cell lines, KRAS, SLC7A11, and Nrf2 are contemporaneous immunoblots that run in parallel from the same biological replicate. (F) SLC7A11 suppression upon genetic depletion of Nrf2. Nrf2-sg1 and Nrf2-sg2 represent 2 individual small guide RNAs targeting Nrf2 for editing. (G and $\mathbf{H})$ SLC7A11 and Nrf2 expression upon blockade of KRAS signaling at the mRNA (G) and protein levels (H). A549 cells were treated with LY294002 ( $1 \mu \mathrm{M}, \mathrm{PI3K}$ inhibitor), afuresertib (5 $\mu \mathrm{M}$, Akt inhibitor), dabrafenib (20 $\mu \mathrm{M}$, Raf inhibitor), AZD6244 (20 nM, MEK inhibitor), and RBC8 (10 $\mu$ M, Ral GTPase inhibitor) for 48 hours, respectively. Results are shown as mean \pm SD of biological triplicates. ${ }^{*} P<0.01$ by unpaired, 2 -tailed Student's $t$ tests. (I) SLC7A11 expression in clinical samples. Scale bars: $50 \mu \mathrm{m}$. (J) Box plots showing SLC7A11 IHC scores. The horizontal lines represent the median; the bottom and top of the boxes represent the 25th and 75th percentiles, respectively; and the vertical bars represent the range of the data. (K) Box plot of SLC7A11 expression in clinical tumors. Stage classification of LUAD refers to the TNM classification. ${ }^{*} P<0.05,{ }^{* *} P<0.01,{ }^{* * *} P<0.001$ by 1-way ANOVA with Tukey's multiple-comparisons test.

primary LUAD samples $(n=51)$ from the General Hospital of Ningxia Medical University, Ningxia, China. Tumor samples were further categorized based on KRAS mutational status as analyzed by direct DNA sequencing. IHC analysis showed that SLC7A11 expression was higher in LUAD compared with adjacent normal lung tissues (Figure 2I). Our bioinformatics analysis (Supplemental Figure 2A), together with other observations (22-24), showed that SLC7A11 was overexpressed in several human cancer types. Importantly, when classifying LUAD samples into KRAS-mutant and WT groups, we found that, strikingly, KRAS-mutant LUAD had a much higher level of SLC7A11 compared with KRAS WT samples (Figure 2J). Furthermore, the increased protein level of SLC7A11 was significantly associated with tumor progression, as indicated by $\mathrm{T}$ staging (Figure 2K). Of note, elevated SLC7A11 gene expression was correlated with the KRAS and NRF2 expression levels in TCGA data sets of human LUAD ( $n=93$; Supplemental Figure $2 \mathrm{~B}$ ). These results collectively suggest that SLC7A11 is overexpressed in KRAS-mutant LUAD and potentially involved in tumor progression in patients. 
A

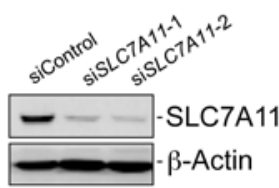

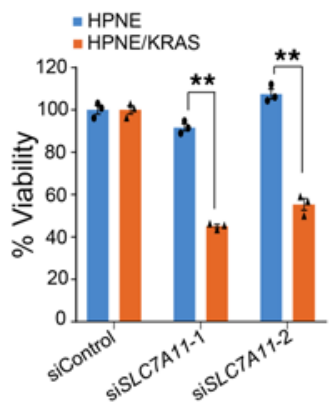
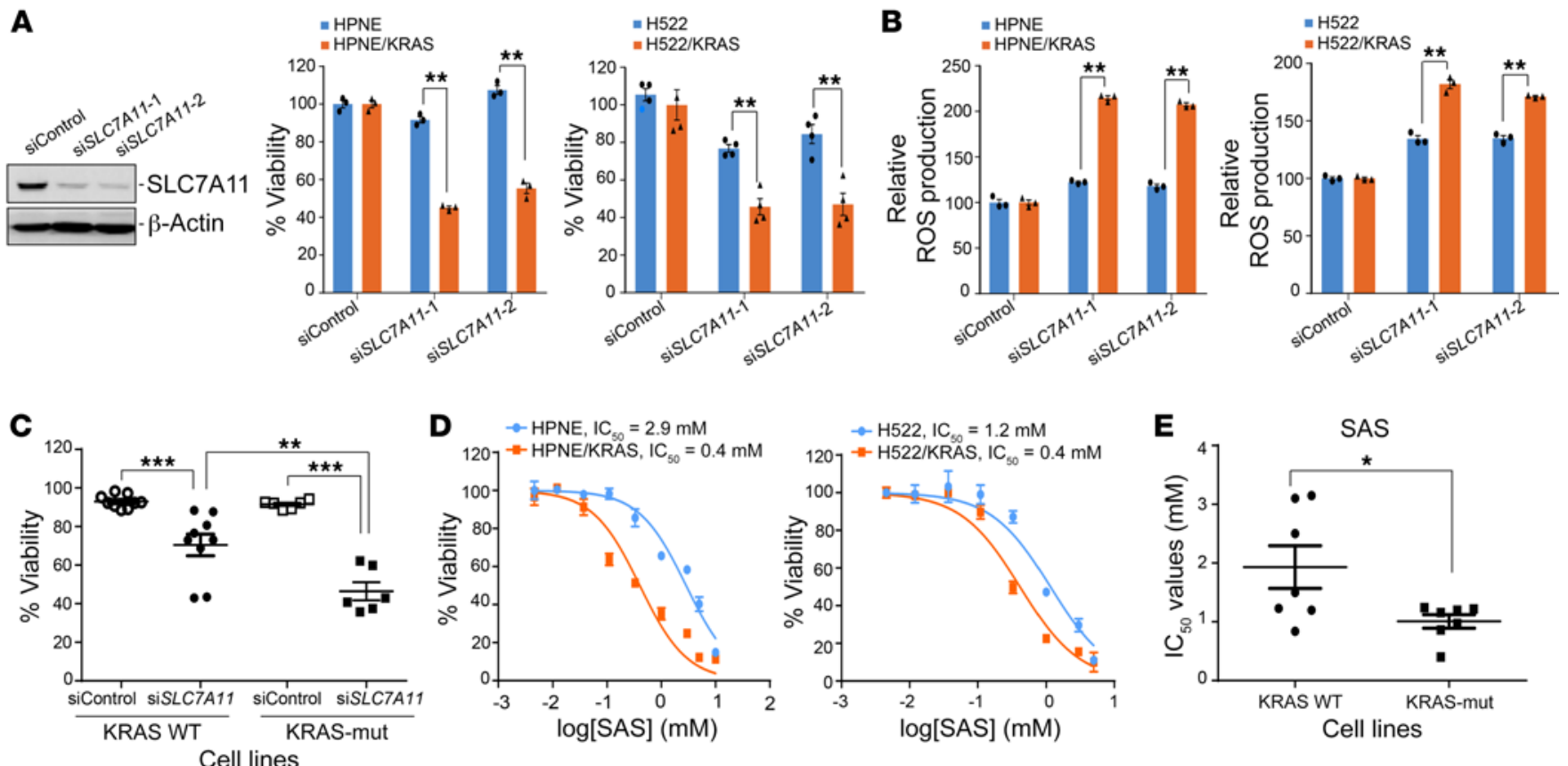

$\mathbf{F}$
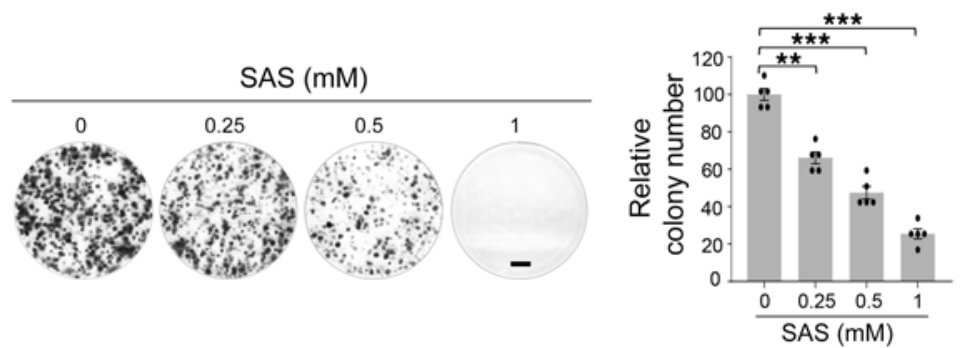

G

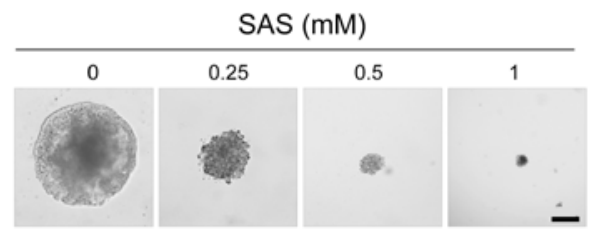

Figure 3. Silencing SLC7A11 selectively kills KRAS-mutant LUAD cells. (A) Effects of SLC7A11 depletion on cell survival. Isogenic cells were transfected with SLC7A11 siRNAs (siSLC7A11) or a scrambled siRNA (siControl). The knockdown efficiency of siSLC7A11 was examined by immunoblotting. Cell viability was measured 72 hours after transfection. Relative cell viability was calculated by setting the values of the siControl-alone group as $100 \%$. (B) Effects of SLC7A11 depletion on ROS production. Relative ROS production was calculated by setting the values of the siControl-alone group as $100 \%$. (C) SLC7A11 depletion led to selective toxicity toward KRAS-mutant cancer cell lines. Six KRAS-mutant and 9 WT cancer cell lines (see Supplemental Table 3) were transfected with siSLC7A11 or a scrambled siRNA. The percent cell viability is relative to the untreated controls. (D) Inhibitory effects of sulfasalazine (SAS) on isogenic cell lines. (E) SAS treatment led to selective toxicity toward KRAS-mutant cancer cells. Seven KRAS-mutant and 7 WT cancer cell lines were treated with SAS for 72 hours. Dots indicate $\mathrm{IC}_{50}$ value of each cell line. (F) Colony formation of A549 cells after SAS treatment. A549 cells were plated in 6-well plates and treated with the indicated concentrations of SAS for 7 days. The relative number of colonies was calculated by normalization to untreated group as $100 \%$. Scale bar: $0.5 \mathrm{~cm}$. (C) Effect of SAS on A549 cell soft agar colony formation. A549 cells were uniformly dispersed in agar and treated with the indicated concentrations of SAS for 21 days. The medium containing SAS was changed twice a week. At the end of the experiment, the colonies were photographed. Scale bar: $2 \mathrm{~mm}$. All data are representative of 3 independent experiments and shown as mean \pm SD of biological triplicates. ${ }^{*} P<0.05,{ }^{* *} P<0.01,{ }^{* *} P<0.001$ by unpaired, 2 -tailed Student's $t$ tests.

As the Keap1/Nrf2 pathway is often altered in LUAD and squamous cell carcinoma of the lung, we next examined Keap1 expression in paired KRAS isogenic pancreatic and lung cell lines (HPNE and HPNE/KRAS; H522 and H522/KRAS). By contrast to elevated Nrf2 expression (Figure 2, A and B), Keap1 levels were little changed in these cell pairs (Supplemental Figure 3). To further query the correlation between SLC7A11 and Nrf2 or Keap1 status, we performed IHC staining to assess Nrf2 and Keap1 expression levels in human LUAD (Supplemental Figure 4, A and B). Our results showed that Nrf2 was overexpressed in human KRASmutant LUAD compared with levels in KRAS WT LUAD or adjacent normal lung tissue. Furthermore, high Nrf2 expression was correlated with high SLC7A11 expression in KRAS-mutant LUAD (Supplemental Figure 4C). However, by contrast, the Keap1 IHC signal did not differ among these groups, and did not show correlation with SLC7A11 expression (Supplemental Figure 4, B and D), indicating that activation of Nrf2 and SLC7A11 might occur via Keap1-independent mechanisms.

Silencing SLC7A11 selectively kills KRAS-mutant LUAD cells. To explore the functional role of SLC7A11 in cell growth, we depleted SLC7A11 in those KRAS isogenic cell lines (Figure 3A). The specificity of the targeting of the siRNAs to SLC7A11 was tested by using C911 oligonucleotides (Supplemental Figure 5). Our results showed that silencing SLC7A11 suppressed cell growth (Figure 3A) and promoted ROS production with high selectivity toward KRASmutant cells, leaving their WT counterparts little affected (Figure 3B). Based on these observations, we further tested sensitivity to SLC7A11 depletion across an additional 32 cancer cell lines (Sup- 
A

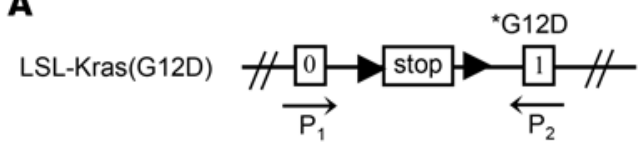

B

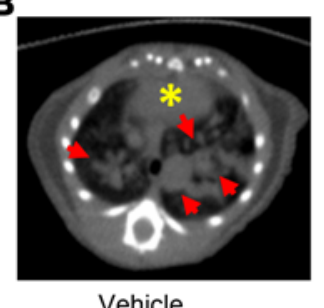

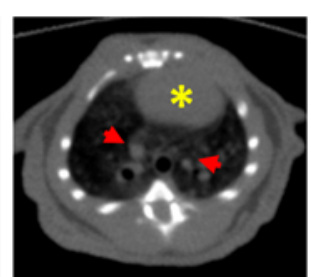

Trametinib $(1 \mathrm{mg} / \mathrm{kg})$

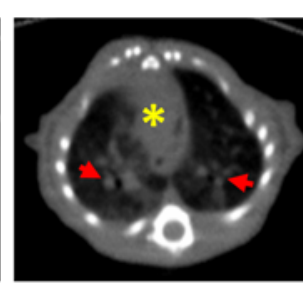

SAS $(250 \mathrm{mg} / \mathrm{kg})$
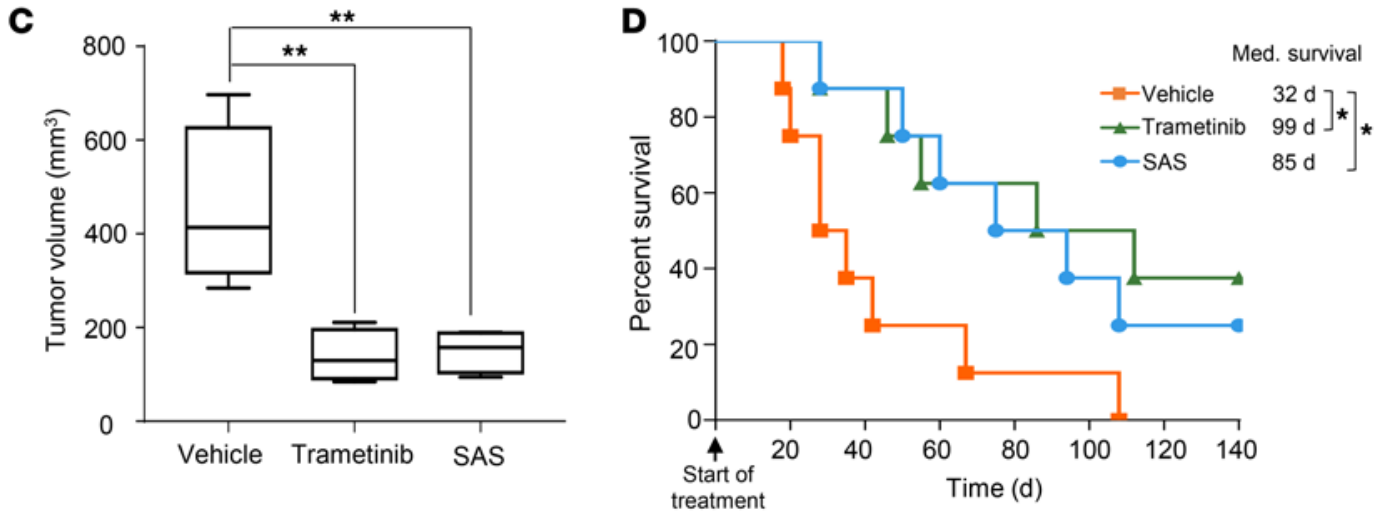

Figure 4. Sulfasalazine leads to tumor regression in vivo. (A) Schematic illustration of the LSL-Kras ${ }^{\mathrm{G} 12 D}$ allele and drug treatment protocol. LSL-Kras ${ }^{\mathrm{G} 120}$ mice were induced with adenovirus-Cre. After a 12 -week induction, mice were treated with vehicle, trametinib (1 mg/kg), or sulfasalazine (250 mg/kg) for an additional 4 weeks. P1 and P2, qPCR forward and reverse primers, respectively. (B) Representative images of tumors from LSL-Kras ${ }^{\mathrm{C} 120}$ mice. Animals were scanned by microCT. Red arrows indicate lung tumors, and yellow asterisks indicate heart. (C) Box plots showing the tumor volumes at the endpoint of the indicated treatments based on microCT $(n=5)$. The horizontal lines representing the median; the bottom and top of the boxes represent the 25th and 75th percentiles, respectively; and the vertical bars represent the range of the data. All data are shown as mean \pm SD. ${ }^{* *} P<0.01$ by 1 -way ANOVA with Tukey's multiple-comparisons test. (D) Kaplan-Meier survival curves for LSL-Kras ${ }^{\mathrm{G} 120}$ mice after the indicated treatments $(n=8)$. Med., median. ${ }^{*} P<0.05$ by log-rank tests.

plemental Table 3). Our results showed that SLC7A11 depletion led to a significant difference in toxicity between KRAS-mutant and WT cell lines (Figure 3C). In attempting to validate SLC7A11 as a mutant KRAS-associated vulnerability, we tested the effects of the SLC7A11 inhibitor sulfasalazine in KRAS experimental systems. Sulfasalazine, an FDA-approved drug, is typically used to treat chronic inflammatory disease and has been shown to decrease the supply of cystine (35). In our paired KRAS isogenic cell lines, we found that KRAS-mutant cells were more vulnerable to sulfasalazine treatment and exhibited much lower $\mathrm{IC}_{50}$ values (Figure 3D). In agreement with this finding, sulfasalazine selectively killed KRAS-mutant compared with KRAS WT cancer cell lines, with much lower $\mathrm{IC}_{50}$ values (Figure 3E). Sulfasalazine also remarkably reduced the growth of A549 cells in long-term clonogenic assays (Figure 3F) and anchorage-independent growth assays (Figure 3G). These results indicate the selectivity and potency of SLC7A11 inhibition toward KRAS-mutant LUAD cells.

Sulfasalazine leads to tumor regression in vivo. In light of the above in vitro findings, we further tested the therapeutic effect of sulfasalazine in the LSL-Kras ${ }^{\mathrm{G} 12 \mathrm{D}}$ mouse model in vivo. After oncogene expression was induced by adenovirus expressing Cre recombinase for 12 weeks, mice were randomly treated with vehicle, trametinib, or sulfasalazine for an additional 4 weeks (Figure 4A). Sulfasalazine at a dose of $250 \mathrm{mg} / \mathrm{kg}$ daily led to marked tumor inhibition (Figure 4, B and C) and produced a significant improvement in overall survival (Figure 4D) compared with vehicle. The efficacy exerted by sulfasalazine was comparable to that of the MEK inhibitor trametinib at a dosage of $1 \mathrm{mg} / \mathrm{kg} / \mathrm{d}$. Taken together, these results suggest that SLC7A11 is essential for the growth of KRAS-mutant LUAD in vivo and targeting SLC7A11 represents a potential therapeutic strategy for the treatment of this disease.

Identification of HG106 as a potent SLC7A11 inhibitor. Sulfasalazine exhibited selective inhibition of KRAS-mutant lung cancer cells while sparing WT counterparts (Figures 3 and 4). However, all current available SLC7A11 inhibitors, including sulfasalazine, have shown low target specificity against SLC7A11 (19). This was also supported by our in vivo data indicating that sulfasalazine produced satisfactory therapeutic efficacy only at high dosages, as much as $250 \mathrm{mg} / \mathrm{kg}$ daily (Figure 4, B and C). High-dosage administration of sulfasalazine probably would result in adverse and off-target effects when used for the treatment of human cancers. Hence, we sought to identify more potent inhibitors of SLC7A11 by screening compounds while monitoring cellular cystine uptake and glutathione biosynthesis through radioactivity and fluorescence, respectively.

A primary screening was carried out on a commercial library based on the compounds' inhibitory effects on glutathione production in A549 cells. The mean value and SD of the experimental population screened were expressed as $Z$ scores. Because the $Z$ score indexes of the majority of the compounds ranged from 2 to -2 , we considered "hits" those with $Z$ scores lower than -3 . Based on this cutoff, we identified 8 compounds that potently 
A<smiles>COc1ccc(-n2nc3ccc(NC(=O)CCl)cc3n2)cc1</smiles>

HG106

MW: 316.74
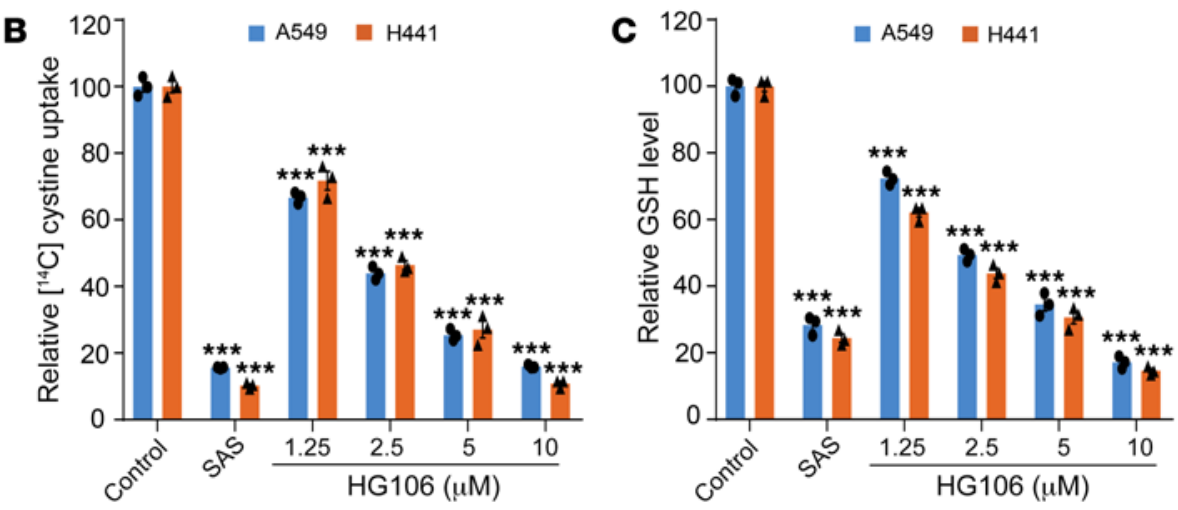

D

Metabolite pathway enrichment

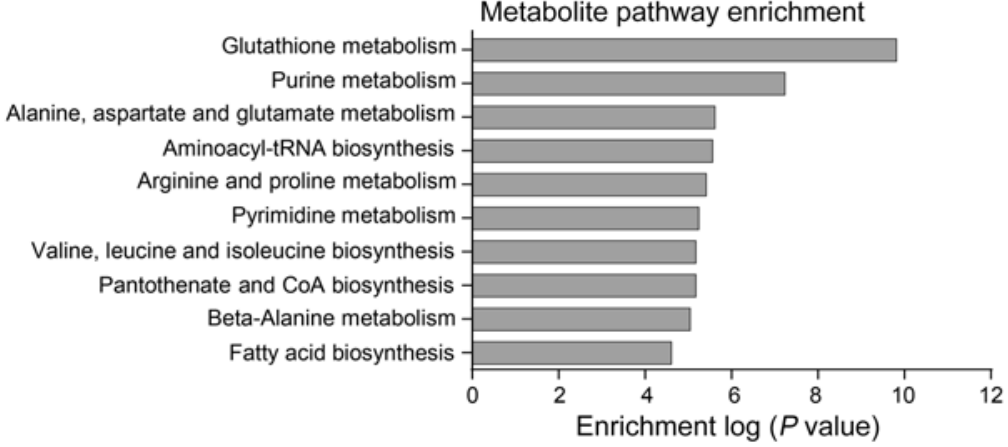

E
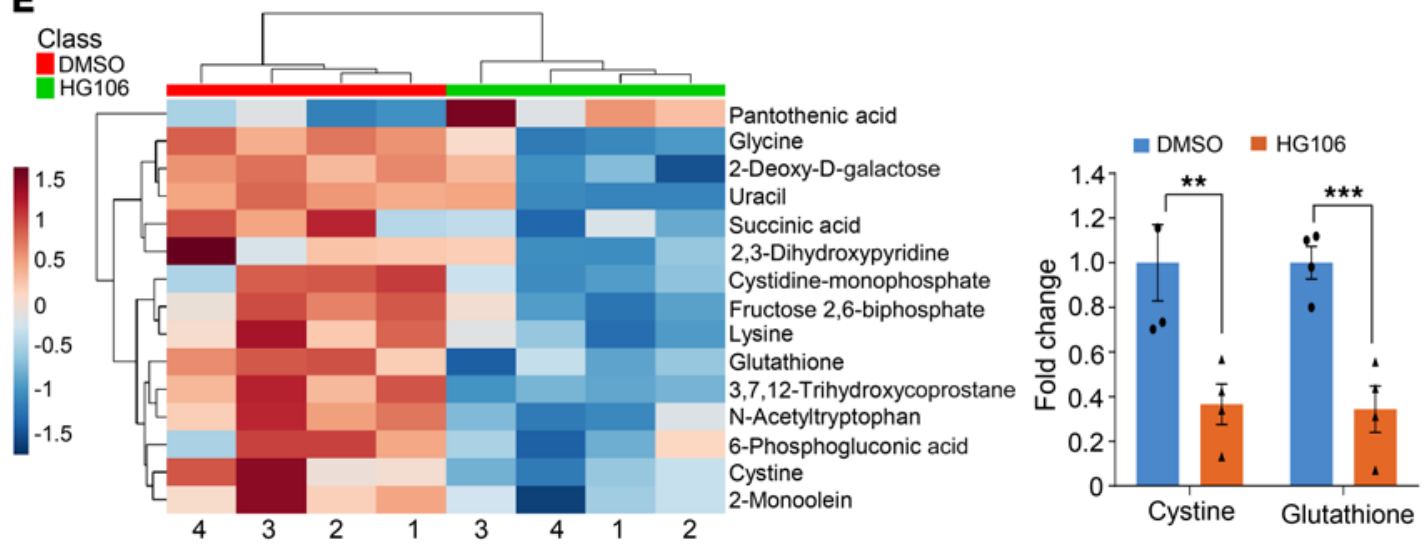

$\mathbf{F}$ H441 KRAS(G12V)

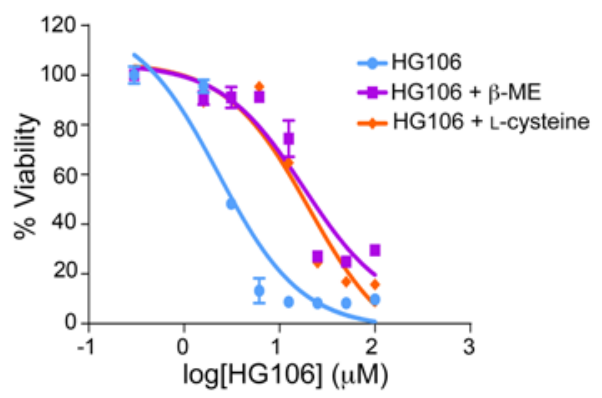

G

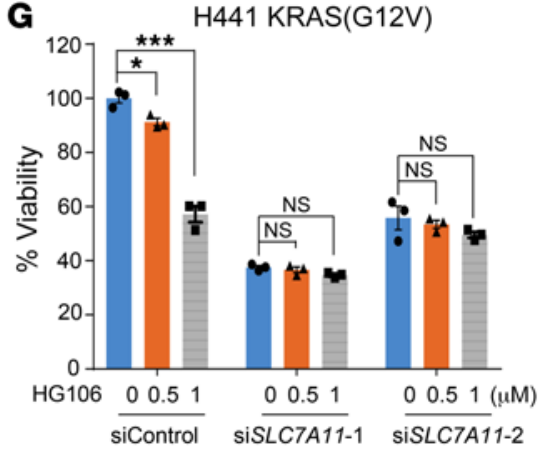


Figure 5. Identification of HG106 as a potent SLC7A11 inhibitor. (A) Chemical structure of HG106. (B and C) HG106 dose-dependently inhibited cystine uptake and GSH level. A549 and H441 cells were treated with HG106 and sulfasalazine (1 mM). Relative cystine uptake and GSH levels were calculated by setting the values of the vehicle control group as $100 \%$. (D) Metabolic pathway enrichment in A549 cells after HG106 treatment. A549 cells were treated with $5 \mu \mathrm{M} \mathrm{HG106}$ for 6 hours. Cell lysates were subjected to metabolomic profiling. For metabolite pathway enrichment analysis, subsets of significantly affected metabolites were chosen. The bar plot shows the top 10 enriched pathways $(n=4)$. (E) Significantly changed metabolites involved in GSH biosynthesis are shown in the heatmap ( $n=$ 4). Changes in cystine and glutathione between the vehicle control- and HG106-treated groups are shown according to the metabolomic data. (F) Effect of $\beta$-mercaptoethanol ( $\beta$-ME, $100 \mu \mathrm{M})$ and L-cysteine $(5 \mathrm{mM})$ on HG106-induced cell death in $\mathrm{H} 441$ cells. $\mathrm{H} 441$ cells were treated with an HC106 concentration gradient with or without $\beta$-ME and L-cysteine for 72 hours, and cell viability was measured. (C) Effect of SLC7A11 knockdown by RNA interference on $\mathrm{HG106-induced} \mathrm{cell} \mathrm{death.} \mathrm{H} 441$ cells were treated with the indicated concentrations of HG106 72 hours after transfection with SLC7A11 siRNAs or a scrambled siRNA. Relative cell viability was calculated by setting the values of the siControl group as $100 \%$. All data are representative of at least 2 independent experiments, and shown as mean \pm SD of biological triplicates. ${ }^{*} P<0.05$, ${ }^{* *} P<0.01$, ${ }^{* *} P<0.001$ by 1-way ANOVA with Tukey's multiple-comparisons test (B, C, and $\mathbf{G}$ ) or by unpaired, 2-tailed Student's $t$ tests (E).

decreased glutathione production, with compound 575148 ranking at the top (Supplemental Figure 6A). Intriguingly, most of these effective compounds belonged to a series of chemicals with a benzotriazole scaffold. Therefore, several derivatives were further synthesized and modified based on the structural properties of compound 575148 (Supplemental Table 4). Those derivatives were subsequently evaluated in a subscreen on cellular glutathione level and cystine uptake. The effects of synthesized derivatives on glutathione production and $\left[{ }^{14} \mathrm{C}\right]$-cystine consumption are shown in Supplemental Figure 6B. The small molecule HG106 (Figure 5A) exhibited the greatest potency and efficacy, and was therefore selected as a candidate for further evaluation against KRAS-mutant LUAD. HG106 inhibited $\left[{ }^{14} \mathrm{C}\right]$-cystine consumption (Figure $5 \mathrm{~B}$ ) and glutathione production (Figure $5 \mathrm{C}$ ) in a concentration-dependent manner in KRAS-mutant LUAD cells, with $1.25 \mu \mathrm{mol} / \mathrm{L}$ being the lowest effective concentration. The efficacy of HG106 at $10 \mu \mathrm{mol} / \mathrm{L}$ was comparable to that of sulfasalazine at a concentration of $1 \mathrm{mmol} / \mathrm{L}$, indicating HG106 had 100-fold-greater activity.

In attempting to confirm and validate the on-target specificity of HG106, we next performed metabolomic profiling to characterize metabolic changes mediated by HG106 treatment. Our results showed that HG106 rewired multiple metabolic pathways, and as expected, glutathione biosynthesis ranked at the top (Figure 5D, Supplemental Table 5, and Supplemental Table 6). Levels of key metabolites in the glutathione metabolism pathway, such as cystine, glutathione, and glycine, were suppressed (Figure 5E and Supplemental Table 7). HG106 exerted substantial cytotoxic effects on $K R A S$-mutant $\mathrm{H} 441$ cells, which were strikingly reduced by addition of $\beta$-mercaptoethanol and L-cysteine (Figure $5 \mathrm{~F}$ ), as they were reported to activate GSH synthesis through a direct increase in cysteine transport $(36,37)$. Moreover, genetic depletion of SLC7A11 significantly reduced the potency of HG106, suggesting its specificity toward SLC7A11 (Figure 5G). Similar results were also observed in A549 cells harboring a G12S mutation in KRAS (Supplemental Figure 7, A and B). HG106 activated ERK, whereas it exhibited no obvious inhibitory actions on a panel of kinases (Supplemental Table 8), RAS activity, or other MAPK pathway components (Supplemental Figure 8).

HG106 preferentially decreases the viability of KRAS-mutant LUAD cells. SLC7A11 promotes cystine uptake and glutathione biosynthesis, leading to protection from oxidative stress (19). We therefore hypothesized that SLC7A11 inhibition by HG106 would lead to increased ROS levels in KRAS-mutant cells. We performed flow cytometry to measure ROS levels. As shown in Figure 6A, HG106 dose-dependently increased total ROS levels in A549 cells. Treatment with the ROS scavenger $N$-acetylcysteine (NAC) markedly reduced the cytotoxicity of HG106 (Figure 6B), supporting a role for ROS in mediating the cytotoxic effects of HG106. In our study, NAC at $10 \mathrm{mM}$ marginally affected the viability of A549 and H441 cells in vitro (Supplemental Figure 9), inconsistent with its tumor-promoting effect in mice (38), suggesting that the impact of antioxidants on tumor cell proliferation might depend on a long-term treatment process.

Oxidative stresses caused by ROS induce rapid depolarization of inner mitochondrial membrane potential (MMP) and subsequent impairment of oxidative phosphorylation, a functional output of the tricarboxylic acid cycle (39). We next examined whether HG106 inhibited mitochondrial oxidative phosphorylation. As shown in Figure 6, C and D, HG106 dose-dependently reduced the oxygen consumption rate and disrupted MMP in A549 and H441 cells. Using transmission electron microscopy, we also observed that cells treated with HG106 exhibited mitochondrial swelling, similar to the effects of hydrogen peroxide (Figure 6E). Accumulation of ROS concomitantly mediated ER stress (40). Our results further showed that HG106 increased the activation of the ER stress-related markers IRE1 $\alpha$, PERK, and GRP78 (Figure $6 \mathrm{~F}$ ), and transcription of $C H O P, A T F 4$, and ATF6 (Supplemental Figure 10). These data suggest that mitochondrial dysfunction and ER stress occur to a marked degree in the presence of HG106 as a consequence of increased intracellular ROS levels.

Oncogenic RAS drives ROS production, and KRAS-mutant cells in turn show susceptibility to ROS manipulation agents (33). We next examined the selective cytotoxicity of HG106 in $K R A S$-mutant cell lines. As expected, HG106 affected viability more in KRAS-mutant cell lines compared with their WT counterparts (Figure 6G). HG106 also selectively killed KRAS-mutant versus WT cells of the abovementioned cancer cell lines (Figure $6 \mathrm{H}$ and Supplemental Table 9). Importantly, normal cells were less affected by HG106 when compared with KRAS-mutant cells (Supplemental Figure 11 and Supplemental Table 9), indicating the safety and low toxicity of HG106. Intriguingly, HG106 treatment did not lead to autophagy-related cell death (Supplemental Figure 12A) or ferroptosis (Supplemental Figure 12B). ROS-mediated mitochondrial dysfunction and elevated ER stress have been implicated in promoting tumor cell apoptosis (41). In accordance with this, HG106 significantly induced apoptosis in KRAS-mutant LUAD cells (Figure 6I) and inhibited colony formation (Figure 6J). All these results suggest that HG106 leads to a lethal increase in ROS levels in KRAS-mutant LUAD cells, causing selective cellular ER stress and apoptosis. 

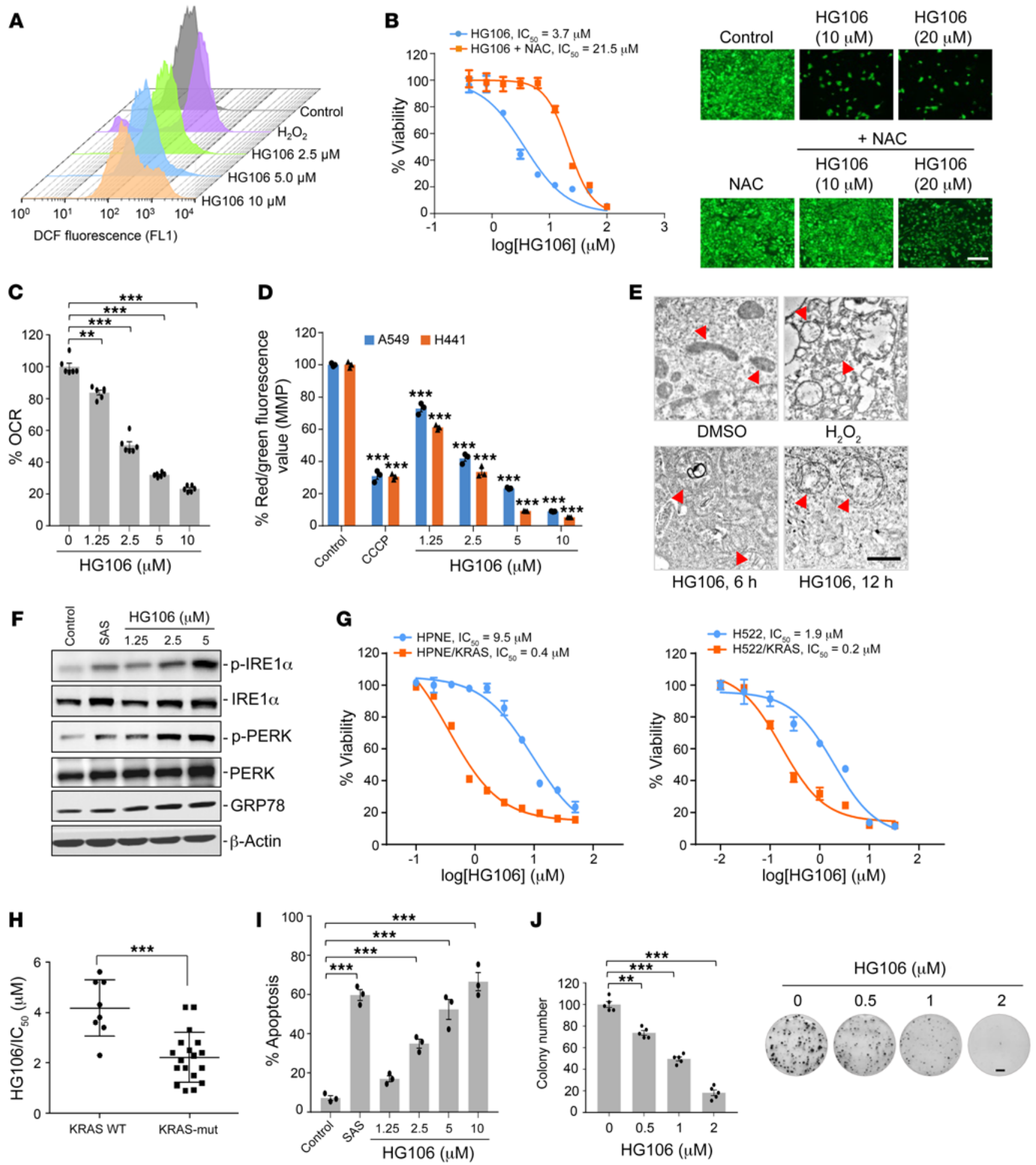

Figure 6. HG106 preferentially decreases the viability of KRAS-mutant LUAD cells. (A) 3D plot showing ROS production. A549 cells were treated with $\mathrm{H}_{2} \mathrm{O}_{2}$ for 1 hour or different concentrations of HG106 for 6 hours. (B) Effect of NAC (10 mM) on HC106-induced cell death. A549 cells were treated with HC106 alone or in combination with NAC for 72 hours, and cell viability was measured. Survival data for the HG106 group and the HC106+NAC group were normalized to the untreated control group and NAC-alone group, respectively. Cell survival was also determined by calcein-AM staining (right; green, viable cells). Scale bar: $50 \mu \mathrm{m}$. (C) Basal mitochondrial oxygen consumption rate (OCR) changes in HC106-treated A549 cells. OCR values were normalized to sulforhodamine staining. (D) MMP change in HC106-treated A549 cells. CCCP, carbonyl cyanide 3-chlorophenylhydrazone. (E) Mitochondria morphology. Red arrowheads indicate swelling of mitochondria. Scale bar: $0.5 \mu \mathrm{m}$. (F) HG106 activated ER stress-related markers. A549 cells were treated with HG106 and sulfasalazine (1 mM) for 24 hours. Immunoblots were contemporaneous and run in parallel from the same biological replicate. (C) Effect of HG106 on cell viability of KRAS isogenic cells. (H) HG106 selective kills KRAS-mutant cancer cells. A panel of KRAS mutant $(n=18)$ and WT KRAS $(n=8)$ cancer cell lines (see Supplemental Table 9$)$ were treated with HG106 for 72 hours. Dots indicate $\mathrm{IC}_{50}$ value of each cell line. (I) A549 cell apoptosis induced by HG106. (J) The effect of HG106 on A549 cell colony formation. Colony number was normalization to the control. Scale bar: $0.5 \mathrm{~cm}$. All data are representative of at least 3 independent experiments and shown as mean \pm SD of biological triplicates. ${ }^{* *} P<0.01,{ }^{* *} P<0.001$ by 1-way ANOVA with Tukey's multiple-comparisons test (C, D, I, and $\left.\mathbf{J}\right)$ or by unpaired, 2-tailed Student's $t$ tests (H). 
In vivo responses of KRAS-mutant LUAD to HG106. Based on the in vitro potency of HG106, we then explored its antitumor activity against KRAS-mutant LUAD in vivo in a series of preclinical mouse models. We first set up a lung cancer cell xenograft mouse model. A549 cells were injected s.c. into the flanks of 4- to 5-week-old male athymic nude mice. When tumors reached a volume about $150 \mathrm{~mm}^{3}$, mice were treated with vehicle chow or different dosages of HG106 through daily intraperitoneal injection. As shown in Figure 7A, continuous HG106 treatment led to prolonged tumor growth inhibition at tested doses. To investigate the HG106 efficacy at a more translational level, we then set up a patient-derived xenograft (PDX) model of LUAD harboring a G12V mutation in KRAS. After 3 weeks of treatment, HG106 strikingly suppressed PDX tumor growth (Figure 7B). Of note, the effect of HG106 therapy was well tolerated, since no animals in any group exhibited systematic toxicity in these studies (Supplemental Figure 13, A and B). HG106 at doses of 2 or $4 \mathrm{mg} / \mathrm{kg}$ markedly increased ROS production and TUNEL signal in patient-derived xenografts (Supplemental Figure 14, A and B), validating that HG106 triggered ER stress-induced apoptosis in vivo.

In attempting to further explore the clinical benefit of HG106 in mouse survival, we carried out efficacy experiments in two conditional genetic models using activation of oncogenic KRAS alone or in combination with loss of function of TRP53. As shown in LSLKras $^{\mathrm{G} 12 \mathrm{D}}$ mice, lung tumor volume (as assessed by microCT imaging) was significantly reduced by HG106 (Supplemental Figure 15 , A and B). The median survival in infected LSL-Kras ${ }^{\mathrm{G} 12 \mathrm{D}}$ vehicle mice was 39 days, whereas it was prolonged to 81 or 106 days in two HG106-treated groups, respectively (Supplemental Figure $14 \mathrm{C})$. Activation of an oncogenic allele of KRAS is sufficient to initiate the tumorigenesis process in lung, whereas additional deletion or point mutation of a tumor suppressor leads to significantly faster development of adenocarcinomas that have features of more advanced disease, similar to that in humans (42). We thus set up the LSL-Kras ${ }^{\mathrm{G} 12 \mathrm{D} /+} ; \operatorname{Trp} 53^{\mathrm{fl} / \mathrm{ll}}$ conditional mouse model, and we found that the LUAD in the vehicle group grew aggressively and diffused quickly into the entire lung tissue over a 1-month period. However, HG106 treatment led to significant tumor inhibition compared with the vehicle group $(P<0.001$; Figure 7, C and D). More encouragingly, HG106 therapy produced a higher long-term survival advantage (log-rank test; $P=0.0048$; Figure 7E). Based on the results from 4 preclinical mouse models, we confirmed that HG106 exhibited potent in vivo efficacy with a satisfactory therapeutic window for the treatment of LUAD.

\section{Discussion}

Approximately $30 \%$ of LUAD tumors carry mutations in KRAS, but no clinically applicable targeted strategy is yet available. To address this challenge, we profiled metabolic dysregulation in isogenic cell pairs with or without KRAS mutation to identify metabolic vulnerabilities for the treatment of KRAS-driven LUAD. We found dramatic changes in metabolites upon KRAS mutational activation, especially intermediates in glutathione biosynthesis. We further identified SLC7A11, which centrally regulates adaptive glutathione metabolism by conferring specificity for cystine uptake, as a synthetic lethal target in KRAS-mutant LUAD (Figure $8 \mathrm{~A})$. In particular, we found that SLC7A11 was highly overex- pressed in KRAS-mutant LUAD cells and tissues, displaying a significant association with tumor progression. Targeting SLC7A11 significantly impaired growth and survival of KRAS-mutant LUAD in vitro and in vivo, indicating that SLC7A11 was a functional and translatable target. More importantly, we developed a potent SLC7A11 inhibitor, HG106, through a function-based chemical screen. SLC7A11 inhibition by HG106 selectively increased metabolic stress- and oxidative stress-mediated apoptosis in LUAD harboring KRAS mutations (Figure $8 \mathrm{~B}$ ). Overall, our findings demonstrate that suppression of the SLC7A11/glutathione axis causes metabolic lethality specifically in KRAS-mutant LUAD. The synthetic lethal link between KRAS mutational status and a requirement for SLC7A11 function may enable promising therapeutic approaches for the treatment of KRAS-mutant cancers.

Previous reports have indicated that KRAS-mutant tumor cells display distinct metabolic requirements compared with their WT counterparts (43). Although these metabolic changes support growth and proliferation, they expose the Achilles' heel of cancer cells. Recently, a synthetic lethal screen carried out in a 3D clonogenic system identified inhibitors of dihydroorotate dehydrogenase that perturbed pyrimidine biosynthesis and selectively inhibited the growth of KRAS-mutant pancreatic cancer (44). SLC25A22, a mitochondrial glutamate transporter, has also been identified as a synthetic lethal partner of KRAS (45). All these studies support the notion that targeting metabolic synthetic lethality may be a promising approach for cancer therapy (16). In our study, we revealed the glutathione dependence of KRAS-mutant cells in comprehensive experiments. We found elevated endogenous glutathione levels coupled with increased cystine uptake in the presence of KRAS mutational activation (Figure 1). Glutathione has a predominant antioxidative function in sustaining redox homeostasis. In response to redox equilibrium imbalance, KRAS mutant cells tend to enhance glutathione-mediated detoxification $(30,31)$. Our results agree with the notion that disrupting redox homeostasis is an effective therapeutic option for KRAS-driven tumors $(4,32)$. Although it has become clear that tumors show varying degrees of reliance on oncogenic KRAS, due to metabolic variations, several aspects of how KRAS dependence impacts metabolic rewiring (e.g., glutathione synthesis and redox homeostasis) and the associated vulnerability in a complex tumor microenvironment remained to be determined.

Targeting of metabolic alterations overactivated in the context of a mutant KRAS is critical. SLC7A11 has well-known functions, serving as the specific antiporter of cystine intake $(22,46)$. SLC7A11 is upregulated in several types of cancer and represents an independent prognostic factor (20-24). In addition, SLC7A11 confers drug resistance by supplying cystine for glutathione maintenance (25-27) and represents an acquired vulnerability of MAPK inhibitor-resistant melanomas (26). Oncogenic KRAS and its effector pathways induce Nrf2, the master transcriptional regulator of endogenous antioxidant synthesis $(34,47)$. Knockdown of KRAS or inhibition of PI3K suppresses Nrf2 activity and further regulates ATF4 (47). Inhibition of the MAPK pathway results in decreased induction of Nrf2 and its target genes via the transcription factors Jun and Myc (34). In our study, we additionally incorporated the Ral GTPase inhibitor RBC8 into experiments and found that it could also lead to Nrf2 suppression, even though its efficacy was 

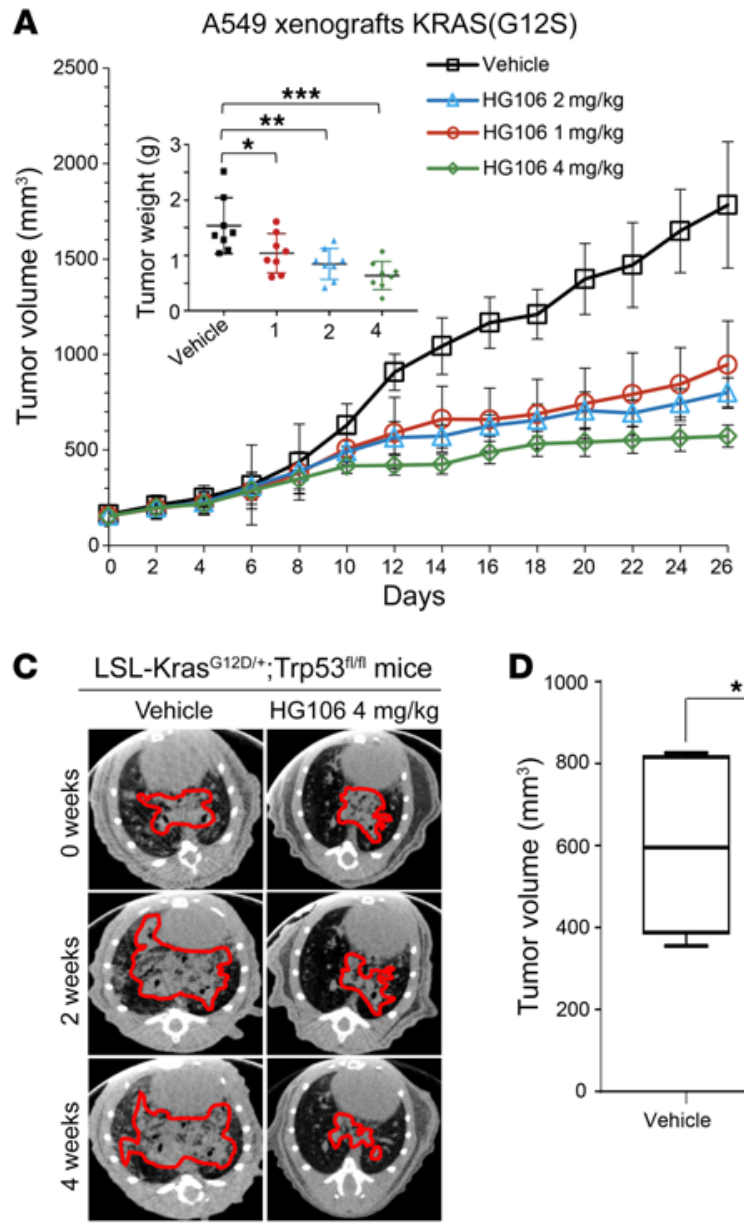

B

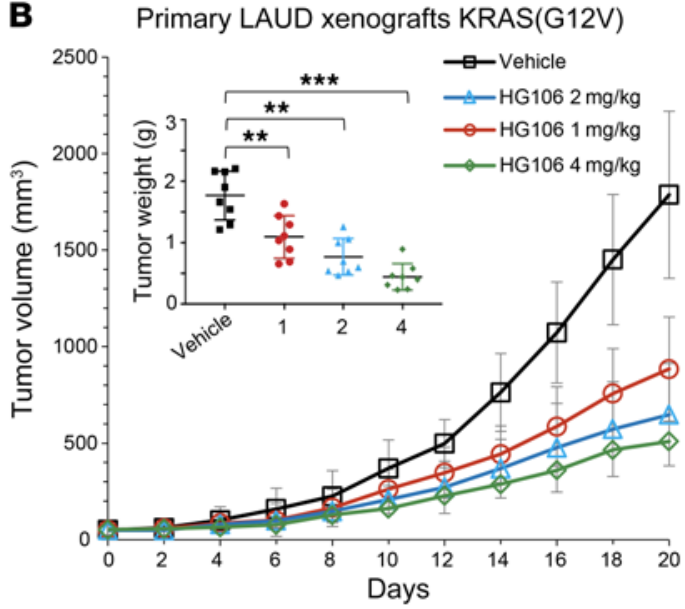

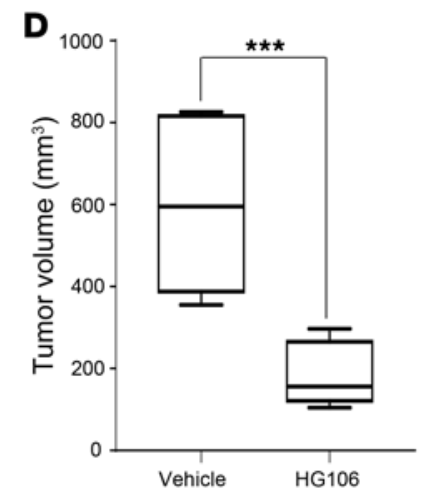

$\mathbf{E}$

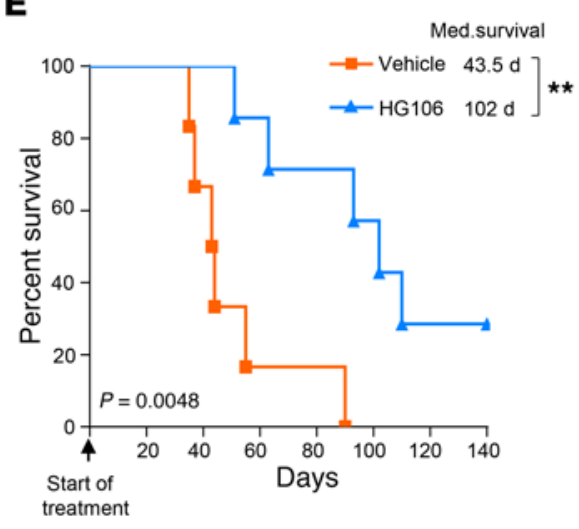

Figure 7. In vivo responses of KRAS-mutant LUAD to HG106. (A) A549 xenograft growth curve $(n=8)$. Mean weights of tumors on day 26 are shown in the inset. (B) Growth curve of patient-derived xenografts $(n=8)$. Mean tumor weight on day 20 is shown in the inset. Values are expressed as mean \pm SEM. ${ }^{*} P<0.05,{ }^{* *} P<0.01,{ }^{* *} P<0.001$ by 1-way ANOVA with Tukey's multiple-comparisons test. (C) Representative tumor images of LSL-Kras ${ }^{\mathrm{G120} /+} ; \operatorname{Trp}^{*} 3^{\mathrm{fl} / \mathrm{fl}}$ (KP) mice. KP mice bearing established tumors were treated with HG106. Animals were scanned by microCT during treatment. (D) Tumor volumes in KP mice $(n=6 \sim 7)$. Tumor volumes at the endpoint of the indicated treatments are shown as box plots. Data are shown as mean \pm SD. The horizontal lines represent the median; the bottom and top of the boxes represent the 25th and 75th percentiles, respectively; and the vertical bars represent the range of the data. ${ }^{* *} P<0.001$ by unpaired, 2 -tailed Student's $t$ tests. (E) Kaplan-Meier survival curves of KP mice $(n=6 \sim 7) .{ }^{*} P<0.01$ by log-rank tests.

lower than that of the MEK inhibitor, suggesting potential cooperativity of KRAS effector pathways in regulating Nrf2 transcription and function. In response to oxidative stress, Nrf2 in turn activates downstream elements including SLC7A11 $(34,48)$. However, the direct connection between SLC7A11 and mutant KRAS has not been clearly identified. Our results showed that mutant KRAS significantly promoted glutathione biosynthesis (Figure 1). In both KRAS isogenic cell lines and human KRAS-mutant LUAD tissues, SLC7A11 abundance was positively correlated with KRAS mutational activation, and this was in step with Nrf2 expression (Figure 2). Genetic disruption of KRAS decreased Nrf2, and key pathways downstream of KRAS shared the ability to modulate the Nrf2/ SLC7A11 axis (Figure 2, E-H). Together with the published work demonstrating that KRAS-directed increase in Nrf2 expression is a mechanism for the activation of the Nrf2 antioxidant program (34), we proposed that Nrf2 might be a target of KRAS. Recently, Lim and colleagues independently revealed that the cystine/glutamate transporter SLC7A11 is essential for KRAS-induced tumorigenicity through enhancement of glutathione synthesis, and identified a synergy mechanism whereby transcription factors of ETS-1 and ATF4 regulate SLC7A11 expression (49). ATF has been reported as both a transcriptional target and heterodimerization partner of Nrf2 $(50,51)$. Since there are cis-acting transcriptional regulatory elements present in the SLC7A11 promoter, it is likely that several transcription factors (such as NRF2 and ATF4) could recognize them and share the ability and potential cooperativity to modulate SLC7A11 expression and function, particularly in cancer cells exhibiting an activated KRAS pathway. The dependence of KRAS-mutant cancers on increased glutathione biosynthesis could be therapeutically exploited through SLC7A11 silencing (Figure 3). Several agents have been characterized to suppress the transport activity of SLC7A11 (36), among which the most promising is an FDA-approved agent, sulfasalazine, that is typically used to treat chronic inflammatory disease (35). In accordance with the results of genetic depletion of SLC7A11, KRAS-mutant cells showed more sensitivity to sulfasalazine as well. Administration of sulfasalazine in the LSL-Kras ${ }^{\mathrm{G} 12 \mathrm{D}}$ mouse model significantly reduced tumor burden (Figure 4), consistent with previous studies about its in vivo 
A

\section{Mutant KRAS-mediated} increase in $\mathrm{GSH}$ biosynthesis

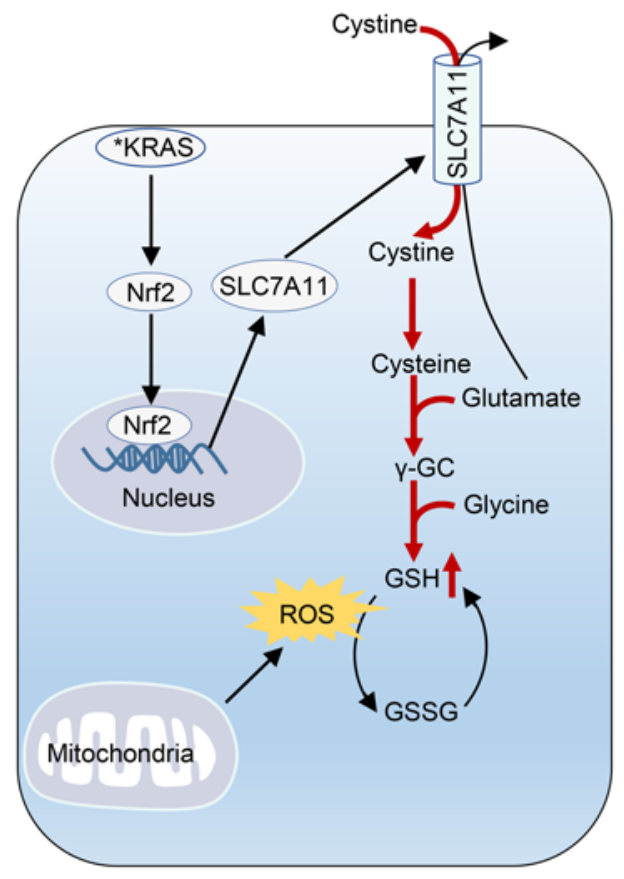

B

Selective killing of KRAS-mutant cancer cells by HG106

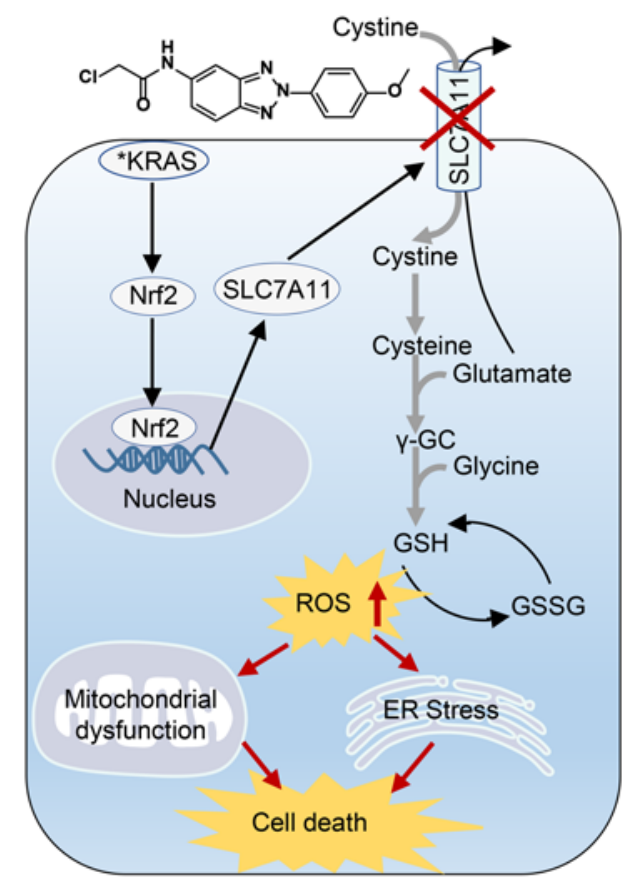

Figure 8. Schematic representation of KRAS-regulated glutathione metabolic reprogramming and the selective killing of KRAS-mutant cells by HG106. (A) Activated KRAS induces SLC7A11 overexpression through activation of transcription factor Nrf2. As a consequence, SLC7A11 takes up a high level of cystine from the extracellular environment to generate more glutathione (GSH), which plays an important role in sustaining the oxidative balance in KRAS-mutant cells. Ultimately, KRAS-mutant cells are highly dependent on SLC7A11-mediated GSH biosynthesis. (B) When treated with HC106, a potent SLC7A11 inhibitor, cellular oxidant-antioxidant homeostasis is severely disrupted, coupled with significant mitochondrial dysfunction and ER stress, ultimately leading to massive cell death of KRAS-mutant cancers. Activated KRAS is indicated by an asterisk. Red arrows indicate pathway activation, and gray arrows indicate pathway inhibition. antitumor activity in other types of cancer (19, 22, 25). Overall, these observations validate that $S L C 7 A 11$ is a synthetic lethal partner of KRAS, providing a direct link between these two factors.

Sulfasalazine inhibits SLC7A11 and leads to in vivo therapeutic effects; however, all current available SLC7A11 inhibitors, including sulfasalazine, have off-target effects, thereby limiting their use as SLC7A11-specific inhibitors in clinical settings (19). This was also evidenced by our results indicating that sulfasalazine led to tumor regression at a substantially high dosage of $250 \mathrm{mg} / \mathrm{kg}$ in LSL-Kras ${ }^{\mathrm{G} 12 \mathrm{D}}$ mice (Figure 4). This prompted us to identify and develop highly potent SLC7A11 inhibitors. In the present study, we showed that HG106 concentration-dependently blocked cystine uptake and glutathione biosynthesis by inhibiting SLC7A11 function. Metabolomic profiling further confirmed that glutathione metabolism was primarily inhibited by HG106, indicating its on-target effects (Figure 5). Specifically, suppression of SLC7A11 by HG106 markedly increased the already-elevated levels of ROS and ER stress in KRAS-mutant cells, and consequently led to their apoptosis (Figure 6). Another genotype-selective compound, erastin, identified by a chemical synthetic lethal screen, has been reported to be toxic to cells expressing RAS(G12V) (52). Further study revealed that the potency and activity of erastin was also associated with cystine depletion and impairment of SLC7A11mediated cystine uptake $(53,54)$. In a way, our compound HG106 and erastin are quite similar in action and mechanism, since they both showed genotype-selective activity toward cells harboring RAS mutation through blocking of SLC7A11. These data from small molecules further validate the synthetic lethal connection of KRAS and SLC7A11. However, differences in compound activity remain. HG106 exhibited selective lethality by inducing cell apoptosis, whereas erastin-treated cells undergo ferroptosis, a newly discovered cell death mechanism (53). This may be explained by the differential chemical structures of the two drugs and diversity in elevated ROS-mediated cell death (55).

Considering the potency of the current available SLC7A11 inhibitors, the potential approach to achieve clinical benefit from SLC7A11 inhibition would be to conduct combination therapies. Several lines of evidence demonstrated a synergistic benefit from combined inhibition of SLC7A11 and other targets. SLC7A11 suppression by HDAC inhibitors could result in active cell death in BRAF inhibitor-resistant cells (26). In addition, it has been reported that suppressors of SLC7A11 strong synergized with mutant p53 reactivators by depleting glutathione and inducing massive apoptosis in TP53-mutant cancer cells (41). In light of all this evidence, it might be promising to combine HG106 with other clinical agents, such as MEK inhibitors or chemotherapeutics, to fundamentally defeat KRAS-mutant LUAD. In summary, we identify a druggable synthetic lethal interaction between SLC7A11 and KRAS, revealing promising therapeutic perspectives for KRAS-mutant LUAD.

\section{Methods}

Detailed protocols are provided in Supplemental Methods.

Cell lines and culture. Immortalized and nontumorigenic pancreatic epithelial HPNE cells and their transformed counterparts HPNE/ KRAS cells were generated as previously reported (28). Briefly, mutant KRAS(G12V) was introduced into HPNE cells through a lentivirus delivery system. The H522/KRAS cells were generated by introduction of a KRAS(G12C) mutation into $\mathrm{H} 522$ cells through an adenoassociated virus (AAV) system (Addgene). In brief, AAV-KRAS(G12C) or corresponding empty vector, together with AAV helper plasmid (Rep/ Cap) and pHGTI-adeno1, were transfected into 293T cells, respectively. Adeno-associated virus was generated, collected, and sequentially used to infect $\mathrm{H} 522$ cells in the presence of $10 \mu \mathrm{g} / \mathrm{mL}$ Polybrene (Millipore). After 48 hours of infection, monoclonal populations harboring 
KRAS(G12C) were purified and selected by puromycin (Sangon Biotech). Active KRAS (KRAS-GTP) in monoclonal cells was pulled down with Raf-1 RBD agarose beads (Millipore) and examined by Western blotting assays. The positive monoclones were further identified using Sanger sequencing (BioSune). Details on cell origins and growth conditions are provided in Supplemental Methods. All of the cell lines were authenticated by short tandem repeat analysis before use.

Metabolomic profiling. HPNE and HPNE/KRAS cells were directly harvested without any treatments. A549 cells were plated and treated with HG106 $(5 \mu \mathrm{M})$ for 6 hours. Cells were collected by scraping, and cell pellets from these two sets of samples were immediately flash frozen in liquid nitrogen and stored at $-80^{\circ} \mathrm{C}$ until extraction. The changes in metabolites were examined by GC/TOF-MS-based metabolomics as previously described (56). See Supplemental Methods for details regarding metabolite profiling and pathway analysis.

$\left[{ }^{14} \mathrm{C}\right]$-cystine uptake. Cells were plated at a density of 100,000 cells per well in 12-well plates. After overnight adherence, cells were treated with sulfasalazine (1 mM, 3 minutes) or $\operatorname{HG106}(1.25,2.5,5,10 \mu \mathrm{M}$, 3 minutes). Cells were then washed with PBS and incubated in prewarmed $\mathrm{Na}^{+}$-free buffer at $37^{\circ} \mathrm{C}$ for 10 minutes. After that, $\mathrm{L}-\left[3,3^{\prime}-{ }^{14} \mathrm{C}\right]-$ cystine $(0.2 \mu \mathrm{Ci} / \mathrm{mL}$; PerkinElmer) was added for 15 minutes. Cells were lysed with $200 \mu \mathrm{L}$ of $0.1 \mathrm{M} \mathrm{NaOH}$ solution, and scintillation fluid was sequentially added into harvested cell lysates. Radioactive $\left[{ }^{14} \mathrm{C}\right]$ counts per minute were obtained using a Beckman Coulter LS 6500 Liquid Scintillation Counter.

Glutathione detection. Cells were plated in 6-well plates at a density of 800,000-1000,000 cells per well. Cells were treated with sulfasalazine (1 mM, 12 hours) or HG106 (1.25, 2.5, 5, $10 \mu \mathrm{M}, 12$ hours). Glutathione levels were evaluated using a GSH/GSSG-Glo assay kit (Promega) following the manufacturer's instructions. Glutathione concentration was calculated from an internal standard curve and normalized to total cell number as determined from parallel plates.

Evaluation of ROS. Isogenic cell pairs were harvested at 48 hours after siSLC7A11 transfection. A549 cells were treated with $\mathrm{H}_{2} \mathrm{O}_{2}(16$ $\mathrm{mM}, 1$ hour) or $\mathrm{HG} 106(2.5,5,10 \mu \mathrm{M}, 6$ hours $)$ in 6-well plates at a density of 300,000-500,000 cells per well. All the cells were harvested in conditioned DMEM containing DCFH-DA ( $25 \mu \mathrm{M}$; MilliporeSigma). After incubation at $37^{\circ} \mathrm{C}$ for 30 minutes, cells were washed with PBS and subjected to flow cytometry (FACSCanto II system, BD).

A549 xenograft, patient-derived LUAD xenograft, and autochthonous murine lung cancer models. C57BL/6 and BALB/cA nude mice were purchased from National Rodent Laboratory Animal Resources (Shanghai, China). LSL-Kras ${ }^{\mathrm{G} 12 \mathrm{D} /+} ; \operatorname{Trp} 53^{\mathrm{t} / \mathrm{ll}}$ (KP; B6.129-Kras $\left.{ }^{\mathrm{tm} 4 \mathrm{Tyj}} \operatorname{Trp} 53^{\mathrm{tm} 1 \mathrm{Brn} / \mathrm{J}}\right)$ mice on a C57BL/6J background were purchased from the Jackson Laboratory. Animals were caged in a laminar airflow cabinet under specific pathogen-free conditions, fed with sterilized food and water, and kept on a 12-hour light/12-hour dark cycle. Briefly, 6-week-old BALB/cA nude mice were used to generate A549 xenograft and patient-derived LUAD xenograft mouse models. For the A549 xenograft mouse model, A549 cells $\left(5 \times 10^{6}\right)$ were injected s.c. into mice. For the human primary LAUD xenograft, fragments from a poorly differentiated LAUD harboring a KRAS (G12V) mutation that were obtained from treatmentnaive patients at the Shanghai Changzheng Hospital (Shanghai, China) were implanted s.c. into mice (57). After inoculation of cells or tissues, mice were monitored until tumor volumes grew to $100-200 \mathrm{~mm}^{3}$. Tumor volume was calculated using the following formula: length $\times$ width $\times$ width $\times 0.52$. After that, the mice were randomized into 4 groups and treated with vehicle or different dosages of HG106 for about 1 month. HG106 was dissolved in $0.5 \%$ sodium carboxymethylcellulose (CMC-Na) and delivered daily by intraperitoneal injections. Mice intraperitoneally injected with $0.5 \%$ CMC-Na served as the vehicle control. Tumor volumes were measured every other day, and mouse body weight was recorded in parallel. After the last day of treatment, tumors were excised and weighed as an indicator of tumor burden.

LSL-Kras ${ }^{\mathrm{G} 12 \mathrm{D} /+} ; \operatorname{Trp}^{\mathrm{ft} / \mathrm{fl}}$ mice were used as an autochthonous model for KRAS-mutant adenocarcinomas in this study. In the KP mouse model, intratracheal delivery of adenovirus expressing Cre induced oncogenic Kras activation in lung airway cells and led to the formation of aggressive adenocarcinomas. In brief, 8-week-old KP mice were anesthetized with isoflurane via a gas chamber. Adeno-Cre (HanBio) at a dose of $2.5 \times 10^{7} \mathrm{PFU}$ in a total volume of $125 \mu \mathrm{L}$ was introduced into mice. Five weeks after virus inhalation, lungs were imaged by a Quantum GX microCT Imaging System (PerkinElmer) in order to confirm tumor formation. After tumor formation, animals were randomized into two groups treated with HG106 at a dosage of $4 \mathrm{mg} / \mathrm{kg} / \mathrm{d}$. HG106 was dissolved in 0.5\% CMC-Na and delivered daily by intraperitoneal injections. In parallel, mice intraperitoneally injected with $0.5 \%$ CMC-Na served as the vehicle control. Multifocal adenocarcinomas in mice were measured during the treatment.

Statistics. Unless otherwise noted, data are expressed as mean \pm SD. One-way ANOVA or 2-tailed Student's $t$ test was used to compare the means among groups. The log-rank test was used to compare Kaplan-Meier survival curves. Statistical tests were performed by using Microsoft Excel or GraphPad Prism Software version 5.0. $P$ values less than 0.05 were considered significant.

Study approval. All animal treatments were performed according to the Guide for the Care and Use of Laboratory Animals (National Academies Press, 2011), and all the protocols were approved by East China Normal University. Human LUAD tissue was obtained from the General Hospital of Ningxia Medical University (Ningxia, China) in accordance with approval from the Ethical Committee of the General Hospital of Ningxia Medical University. Prior written informed consent was obtained from patients.

\section{Author contributions}

$\mathrm{KH}, \mathrm{YC}, \mathrm{ML}$, and XP designed and supervised the research. $\mathrm{KH}$, KL, JL, JC, HW, WJ, JW, HP, and ZC performed research and provided helpful discussions. KH, KL, JL, JF, JC, HW, FC, WJ, JW, HP, $\mathrm{ZC}, \mathrm{YC}, \mathrm{ML}$, and XP analyzed and interpreted the data. JF and FC developed the data clustering and performed bioinformatics analysis. JC collected patient samples and conducted pathology evaluations. HW, HP, and YC conducted chemical synthesis. PJC generated and provided KRAS isogenic cell lines. $\mathrm{KH}, \mathrm{KL}$, JL, and XP wrote the manuscript. All authors reviewed and edited the manuscript. $\mathrm{KH}, \mathrm{KL}$, and JL contributed equally as first authors. The order of co-first authors was based on contribution. $\mathrm{KH}$ contributed to all aspects of the study. KL performed animal model experiments and evaluated in vivo efficacy of drugs. JL had a primary role in interpreting and organizing the data as well as writing the manuscript.

\section{Acknowledgments}

We thank Hongbin Ji (Shanghai Institute of Biochemistry and Cell Biology, Chinese Academy of Sciences) for helping set up KP mouse models. We thank Stefan Siwko (Texas A\&M University Health Sci- 
ence Center, Institute of Biosciences and Technology) for manuscript editing. This work is sponsored by the Shanghai Pujiang Program (18PJD014 to XP), National Natural Science Foundation of China (81672758 and 81874207 to XP; 81830083 to ML; 81673304 to YC), Innovation Program of Shanghai Municipal Education Commission (2017-01-07-00-05-E00011 to ML), and the Fundamental Research Funds for the Central Universities (40500-20101-222141 to XP).
Address correspondence to: Xiufeng Pang, Mingyao Liu, or Yihua Chen, Institute of Biomedical Sciences and School of Life Sciences, East China Normal University, 500 Dongchuan Road, Shanghai 200241, China. Phone: 86.21.24206942; Email: xfpang@ bio.ecnu.edu.cn (X. Peng). Phone: 86.21.54345014; Email: myliu@bio.ecnu.edu.cn (M. Liu). Phone: 86.21.24206647; Email: yhchen@bio.ecnu.edu.cn (Y. Chen).
1. Simanshu DK, Nissley DV, McCormick F. RAS proteins and their regulators in human disease. Cell. 2017;170(1):17-33.

2. Rotow J, Bivona TG. Understanding and targeting resistance mechanisms in NSCLC. Nat Rev Cancer. 2017;17(11):637-658.

3. Kris MG, et al. Using multiplexed assays of oncogenic drivers in lung cancers to select targeted drugs. JAMA. 2014;311(19):1998-2006.

4. Cox AD, Fesik SW, Kimmelman AC, Luo J, Der CJ. Drugging the undruggable RAS: mission possible? Nat Rev Drug Discov. 2014;13(11):828-851.

5. Cancer Genome Atlas Research Network. Comprehensive molecular profiling of lung adenocarcinoma. Nature. 2014;511(7511):543-550.

6. Janes MR, et al. Targeting KRAS mutant cancers with a covalent $\mathrm{G} 12 \mathrm{C}$-specific inhibitor. Cell. 2018;172(3):578-589.e17.

7. [No authors listed]. AMG 510 first to inhibit "undruggable" KRAS. Cancer Discov. 2019;9(8):988-989.

8. Hallin J, et al. The KRAS ${ }^{\mathrm{G} 12 \mathrm{C}}$ inhibitor MRTX849 Provides insight toward therapeutic susceptibility of KRAS-mutant cancers in mouse models and patients. Cancer Discov. 2020;10(1):54-71.

9. Canon J, et al. The clinical KRAS(G12C) inhibitor AMG 510 drives anti-tumour immunity. Nature. 2019;575(7781):217-223.

10. Samatar AA, Poulikakos PI. Targeting RAS-ERK signalling in cancer: promises and challenges. Nat Rev Drug Discov. 2014;13(12):928-942.

11. Jänne PA, et al. Selumetinib plus docetaxel compared with docetaxel alone and progressionfree survival in patients with KRASmutant advanced non-small cell lung cancer: the SELECT-1 Randomized Clinical Trial. JAMA. 2017;317(18):1844-1853.

12. Turke AB, et al. MEK inhibition leads to PI3K/AKT activation by relieving a negative feedback on ERBB receptors. Cancer Res. 2012;72(13):3228-3237.

13. Ambrogio C, et al. KRAS dimerization impacts mek inhibitor sensitivity and oncogenic activity of mutant KRAS. Cell. 2018;172(4):857-868.e15.

14. Lv J, Wang J, Chang S, Liu M, Pang X. The greedy nature of mutant RAS: a boon for drug discovery targeting cancer metabolism? Acta Biochim Biophys Sin (Shanghai). 2016;48(1):17-26.

15. Kerr EM, Martins CP. Metabolic rewiring in mutant Kras lung cancer. FEBS J. 2018;285(1):28-41.

16. Zecchini V, Frezza C. Metabolic synthetic lethality in cancer therapy. Biochim Biophys Acta Bioenerg. 2017;1858(8):723-731.

17. Ying H, et al. Oncogenic Kras maintains pancreatic tumors through regulation of anabolic glucose metabolism. Cell. 2012;149(3):656-670.

18 . Son J, et al. Glutamine supports pancreatic cancer growth through a KRAS-regulated metabolic pathway. Nature. 2013;496(7443):101-105.

19. Koppula P, Zhang Y, Zhuang L, Gan B. Amino acid transporter SLC7A11/xCT at the crossroads of regulating redox homeostasis and nutrient dependency of cancer. Cancer Commun (Lond). 2018;38(1):12 .

20. Savaskan NE, et al. Small interfering RNA-mediated $\mathrm{xCT}$ silencing in gliomas inhibits neurodegeneration and alleviates brain edema. Nat Med. 2008;14(6):629-632.

21. Zhang L, et al. Overexpression of SLC7A11: a novel oncogene and an indicator of unfavorable prognosis for liver carcinoma. Future Oncol. 2018;14(10):927-936.

22. Timmerman LA, et al. Glutamine sensitivity analysis identifies the $\mathrm{xCT}$ antiporter as a common triple-negative breast tumor therapeutic target. Cancer Cell. 2013;24(4):450-465.

23. Ji X, et al. xCT (SLC7A11)-mediated metabolic reprogramming promotes non-small cell lung cancer progression. Oncogene. 2018;37(36):5007-5019.

24. Robert SM, et al. SLC7A11 expression is associated with seizures and predicts poor survival in patients with malignant glioma. Sci Transl Med. 2015;7(289):289ra86.

25. Huang Y, Dai Z, Barbacioru C, Sadée W. Cystineglutamate transporter SLC7A11 in cancer chemosensitivity and chemoresistance. Cancer Res. 2005;65(16):7446-7454.

26. Wang L, et al. An acquired vulnerability of drug-resistant melanoma with therapeutic potential. Cell. 2018;173(6):1413-1425.e14.

27. Lo M, Ling V, Wang YZ, Gout PW. The xccystine/glutamate antiporter: a mediator of pancreatic cancer growth with a role in drug resistance. Br JCancer. 2008;99(3):464-472.

28. Chang Z, et al. Cooperativity of oncogenic K-ras and downregulated p16/INK4A in human pancreatic tumorigenesis. PLoS One. 2014;9(7):e101452.

29. Miyanishi K, et al. Glutathione S-transferase-pi overexpression is closely associated with K-ras mutation during human colon carcinogenesis. Gastroenterology. 2001;121(4):865-874.

30. De Raedt T, et al. Exploiting cancer cell vulnerabilities to develop a combination therapy for rasdriven tumors. Cancer Cell. 2011;20(3):400-413.

31. Kerr EM, Gaude E, Turrell FK, Frezza C, Martins CP. Mutant Kras copy number defines metabolic reprogramming and therapeutic susceptibilities. Nature. 2016;531(7592):110-113.

32. Kawamura T, Kondoh Y, Muroi M, Kawatani M, Osada H. A small molecule that induces reactive oxygen species via cellular glutathione depletion. Biochem J. 2014;463(1):53-63.

33. Weinberg F, et al. Mitochondrial metabolism and ROS generation are essential for Krasmediated tumorigenicity. Proc Natl Acad Sci US A.
2010;107(19):8788-8793

34. DeNicola GM, et al. Oncogene-induced Nrf2 transcription promotes ROS detoxification and tumorigenesis. Nature. 2011;475(7354):106-109.

35. Gout PW, Buckley AR, Simms CR, Bruchovsky N. Sulfasalazine, a potent suppressor of lymphoma growth by inhibition of the $\mathrm{x}(\mathrm{c})$ - cystine transporter: a new action for an old drug. Leukemia. 2001;15(10):1633-1640.

36. Dixon SJ, et al. Ferroptosis: an iron-dependent form of nonapoptotic cell death. Cell. 2012;149(5):1060-1072.

37. Ishii T, Bannai S, Sugita Y. Mechanism of growth stimulation of L1210 cells by 2-mercaptoethanol in vitro. Role of the mixed disulfide of 2-mercaptoethanol and cysteine. J Biol Chem. 1981;256(23):12387-12392.

38. Sayin VI, Ibrahim MX, Larsson E, Nilsson JA, Lindahl P, Bergo MO. Antioxidants accelerate lung cancer progression in mice. Sci Transl Med. 2014;6(221):221ra15.

39. Hu Y, et al. K-ras(G12V) transformation leads to mitochondrial dysfunction and a metabolic switch from oxidative phosphorylation to glycolysis. Cell Res. 2012;22(2):399-412.

40. Verfaillie T, et al. PERK is required at the ERmitochondrial contact sites to convey apoptosis after ROS-based ER stress. Cell Death Differ. 2012;19(11):1880-1891.

41. Liu DS, et al. Inhibiting the system $\mathrm{x}_{\mathrm{C}}$-/glutathione axis selectively targets cancers with mutant-p53 accumulation. Nat Commun. 2017;8:14844.

42. DuPage M, Dooley AL, Jacks T. Conditional mouse lung cancer models using adenoviral or lentiviral delivery of Cre recombinase. Nat Protoc. 2009;4(7):1064-1072.

43. Kimmelman AC. Metabolic dependencies in RAS-driven cancers. Clin Cancer Res. 2015;21(8):1828-1834.

44. Koundinya M, et al. Dependence on the pyrimidine biosynthetic enzyme DHODH is a synthetic lethal vulnerability in mutant KRAS-driven cancers. Cell Chem Biol. 2018;25(6):705-717.e11.

45. Wong CC, et al. SLC25A22 promotes proliferation and survival of colorectal cancer cells with KRAS mutations and xenograft tumor progression in mice via intracellular synthesis of aspartate. Gastroenterology. 2016;151(5):945-960.e6.

46. Sato H, Tamba M, Ishii T, Bannai S. Cloning and expression of a plasma membrane cystine/glutamate exchange transporter composed of two distinct proteins. J Biol Chem. 1999;274(17):11455-11458.

47. Gwinn DM, et al. Oncogenic KRAS regulates amino acid homeostasis and asparagine biosynthesis via ATF 4 and alters sensitivity to L-asparaginase. Cancer Cell. 2018;33(1):91-107.e6. 
48. Chen D, et al. NRF2 is a major target of ARF in p53-independent tumor suppression. Mol Cell. 2017;68(1):224-232.e4.

49. Lim JKM, et al. Cystine/glutamate antiporter xCT (SLC7A11) facilitates oncogenic RAS transformation by preserving intracellular redox balance. Proc Natl Acad Sci U S A. 2019;116(19):9433-9442.

50 . $\mathrm{He} \mathrm{CH}$, et al. Identification of activating transcription factor 4 (ATF4) as an Nrf2-interacting protein. Implication for heme oxygenase-1 gene regulation. J Biol Chem. 2001;276(24):20858-20865.

51. Ye P, et al. Nrf2- and ATF4-dependent upregulation of XCT modulates the sensitivity of T24 bladder carcinoma cells to proteasome inhibition. Mol Cell Biol. 2014;34(18):3421-3434.

52. Dolma S, Lessnick SL, Hahn WC, Stockwell BR. Identification of genotype-selective antitumor agents using synthetic lethal chemical screening in engineered human tumor cells. Cancer Cell. 2003;3(3):285-296.

53. Stockwell BR, et al. Ferroptosis: a regulated cell death nexus linking metabolism, redox biology, and disease. Cell. 2017;171(2):273-285.

54. Dixon SJ, et al. Pharmacological inhibition of cystine-glutamate exchange induces endoplasmic reticulum stress and ferroptosis. Elife. 2014;3:e02523.

55. Galluzzi L, et al. Molecular mechanisms of cell death: recommendations of the Nomenclature Committee on Cell Death 2018. Cell Death Differ. 2018;25(3):486-541.

56. Kind T, et al. FiehnLib: mass spectral and retention index libraries for metabolomics based on quadrupole and time-of-flight gas chromatography/mass spectrometry. Anal Chem. 2009;81(24):10038-10048.

57. Wang J, et al. Suppression of KRas-mutant cancer through the combined inhibition of KRAS with PLK1 and ROCK. Nat Commun. 2016;7:11363. 